OPEN ACCESS

Edited by:

Mahendra Dashrath Shirsat,

Dr. Babasaheb Ambedkar

Marathwada University, India

Reviewed by:

Xiaoji Xie,

Nanjing Tech University, China Arshad Saleem Bhatti, COMSATS Institute of Information Technology, Pakistan

*Correspondence:

Wee Shong Chin chmcws@nus.edu.sg

Jianwei XU

jw-xu@imre.a-star.edu.sg

Specialty section: This article was submitted to

Nanoscience,

a section of the journal

Frontiers in Chemistry

Received: 15 July 2019 Accepted: 03 December 2019

Published: 08 January 2020

Citation:

Yemata TA, Zheng Y, Kyaw AKK Wang $X$, Song J, Chin WS and Xu J

(2020) Improved Thermoelectric

Properties and Environmental Stability of Conducting PEDOT:PSS Films

Post-treated With Imidazolium Ionic

Liquids. Front. Chem. 7:870

doi: 10.3389/fchem.2019.00870

\section{Improved Thermoelectric Properties and Environmental Stability of Conducting PEDOT:PSS Films Post-treated With Imidazolium Ionic Liquids}

\author{
Temesgen Atnafu Yemata ${ }^{1,2}$, Yun Zheng ${ }^{1}$, Aung Ko Ko Kyaw ${ }^{1,3}$, Xizu Wang ${ }^{1}$, Jing Song ${ }^{1}$, \\ Wee Shong Chin ${ }^{2 *}$ and Jianwei $\mathrm{Xu}^{1,2 *}$
}

${ }^{1}$ Institute of Materials Research and Engineering, Agency for Science, Technology, and Research (A*STAR), Singapore, Singapore, ${ }^{2}$ Department of Chemistry, National University of Singapore, Singapore, Singapore, ${ }^{3}$ Department of Electrical and Electronic Engineering, Southern University of Science and Technology, Shenzhen, China

Poly(3,4-ethylenedioxythiophene):poly(styrenesulfonate) (PEDOT:PSS) is one of the most popular conducting polymers and widely used as polymer thermoelectric materials, and its thermoelectric performance could be improved by a variety of post-treatment processes. This paper reported two series of post-treatment methods to enhance the thermoelectric performance. The first series method included pre-treatment of PEDOT:PSS film with formamide, followed by imidazolium-based ionic liquids. The second series method included pre-treatment of PEDOT:PSS film with formamide, followed by sodium formaldehyde sulfoxylate, and finally imidazolium-based ionic liquids. Two series of post-treatment methods significantly improved the power factor of PEDOT:PSS when compared to that of PEDOT:PSS treated with formamide only. For example, using the first series post-treatment method with 40 vol.\% ionic liquid 1-butyl3-methylimidazolium bis(trifluoromethanesulfonyl) amide, the Seebeck coefficient of the PEDOT:PSS film increased from 14.9 to $28.5 \mu \mathrm{V} / \mathrm{K}$ although the electrical conductivity reduced from 2,873 to $1,701 \mathrm{~S} / \mathrm{cm}$, resulting in a substantial improvement in the overall power factor from 63.6 to $137.8 \mu \mathrm{W} / \mathrm{K}^{2} \mathrm{~m}$. The electrical conductivity enhancement in the formamide-treatment process was in part ascribed to the removal of the insulating PSS component. Further treatment of PEDOT:PSS film with ionic liquid caused dedoping of PEDOT and hence increased in Seebeck coefficient. In contrast, second series posttreatment method led to the reduction in electrical conductivity from 2,873 to $641 \mathrm{~S} / \mathrm{cm}$ but a big improvement in the Seebeck coefficient from 14.9 to $61.1 \mu \mathrm{V} / \mathrm{K}$ and thus the overall power factor reached up to $\sim 239.2 \mu \mathrm{W} / \mathrm{K}^{2} \mathrm{~m}$. Apart from the improvement in electrical conductivity, the increase in Seebeck coefficient is on account of the substantial dedoping of PEDOT polymer to its neutral form and thus leads to the big improvement of its Seebeck coefficient. The environmental stability of ionic liquid-treated PEDOT:PSS films were examined. It was found that the ionic liquid treated PEDOT:PSS retained 
more than $70 \%$ Seebeck coefficient and electrical conductivity at $75 \% \mathrm{RH}$ humidity and $70^{\circ} \mathrm{C}$ for $480 \mathrm{~h}$. The improved long-term TE stability is attributed to the strong ionic interaction between sulfonate anions and bulky imidazolium cations that effectively block the penetration of water and lessen the tendency to take up water from the air.

Keywords: conducting polymer, PEDOT:PSS, thermoelectric, ionic liquids, environmental stability

\section{INTRODUCTION}

Thermoelectric (TE) materials are able to directly convert heat into electricity and vice versa (Bell, 2008; Snyder and Toberer, 2011). TE devices have been thought as promising "green" power generators and they play an essential role in harvesting lowgrade heat like waste heat or exhausted heat that is usually dissipated to the environment. The performance of TE materials largely depends on the dimensionless figure-of-merit $(Z T), Z T=$ $\sigma S^{2} T / \kappa$ where $\sigma$ is electrical conductivity, $S$ is Seebeck coefficient, $T$ is absolute temperature, and $\kappa$ is thermal conductivity (Snyder and Toberer, 2011). More recently, traditional inorganic materials such as SnSe (Chang et al., 2018; Lee et al., 2019), PbTe (Tan et al., 2016; Chen et al., 2017), GeTe (Li et al., 2018), and $\mathrm{Cu}_{2} \mathrm{Se}_{0.5} \mathrm{~S}_{0.5}$ (Ren, 2017) with $Z T$ values of over 2 have been reported. Despite their impressive TE performance, the drawbacks such as high cost, scarcity, toxicity, and low processability limit their commercial applications. Moreover, most of these promising inorganic TE materials have good performance when operating temperatures exceed $300-400^{\circ} \mathrm{C}$. They may not function well for ambient temperature heat recovery as a large amount of waste heat in our surroundings is below $200^{\circ} \mathrm{C}$ (Yoo et al., 2015). Therefore, organic TE materials with high performance at ambient temperature have gained increasing interest in recovering a huge amount of low temperature waste heat (Yoo et al., 2015).

Conductive polymers (CPs) have immerged as promising TE materials due to the tunable $\sigma$, low $\kappa$, and relatively low production cost. The commonly studied CPs include polyaniline (PANi) (Yoon et al., 1995; Mateeva et al., 1998; MacDiarmid, 2001), poly(3,4-ethylenedioxythiophene):poly(styrenesulfonate) (PEDOT:PSS) (Zhang et al., 2010), polypyrrole (PPy) (Kemp et al., 1999), polyacetylene (PAc) (Kaneko et al., 1993), and polythiophene (PTH) (MacDiarmid, 2001; Hu et al., 2013). Other CPs, like uniaxially aligned iodine $\left(\mathrm{I}_{2}\right)$-doped PAc, has a power factor $\left(P F=S^{2} \sigma\right)$ of $\sim 1,350 \mu \mathrm{W} / \mathrm{K}^{2} \mathrm{~m}$, but it has no practical application due to its poor stability and processability (Cowen et al., 2017). On the contrary, PPy and PANi have good stability but poor PFs of less than $10 \mu \mathrm{W} / \mathrm{K}^{2} \mathrm{~m}$ (Li et al., 2010; Liang et al., 2017). Currently, the TE performance of CPs cannot rival that of state-of-the-art inorganic counterparts. However, the TE performance of CPs has been improved significantly over the years, showing very promising $Z T$ values for applications.

Recently, among the CPs, PEDOT:PSS has attracted great attention due to the high $\sigma$, intrinsically low $\kappa$, waterprocessability, and commercial availability (Khan et al., 2015; Wei et al., 2015). Several approaches have been widely investigated to enhance the TE performance of PEDOT:PSS, such as post treatment (Fan et al., 2017), electrochemical oxidation (Park et al., 2013), and hybrid approach (Zhang et al., 2010), etc. PEDOT:PSS can form hybrids with different carbon sources, metallic nanomaterials or inorganic TE materials. This allows hybrid materials to tap on the advantages of each component, such as a large $S$ or $\sigma$ to balance individual TE characteristics to give an optimum TE performance (Zhang et al., 2010; Moriarty et al., 2013; Xu et al., 2013; Park et al., 2014a). The other challenge is the incompatibility of the two components (i.e., PEDOT:PSS and additives) as observed in many cases, where phase separation of a single component leads to non-uniform films. Surface modification through post-treatment (Mcgrail et al., 2015; Wei et al., 2015) has also proven effective in improving the TE performance of PEDOT:PSS films by removing the insulating PSS segment from PEDOT:PSS. The typical process is to immerse PEDOT:PSS film in dimethyl sulfoxide (DMSO), ethylene glycol (EG) (Culebras et al., 2014; Park et al., 2014b) or inorganic acid solution. Inorganic salts and organic solvents with high dielectric constants have demonstrated the ability in increasing the $\sigma$ of PEDOT by a few orders of magnitude, giving rise to a significant enhancement in $P F$ (Zhang et al., 2010; Culebras et al., 2014). The highest $P F$ can reach up to $469 \mu \mathrm{W} / \mathrm{K}^{2} \mathrm{~m}$, leading to a large $Z T$ value of 0.42 at $300 \mathrm{~K}$ (Kim et al., 2013). These post-treatments can increase the concentration of charge carriers and bipolarons. However, the high doping level in general results in a small $S$ because of the extra charge carriers (Park et al., 2014b). Therefore, appropriate methods that improve $S$ are desired as the $Z T$ involves the square of $S$. Previous studies already demonstrated that the $P F$ could be improved by regulating the redox level through an electrochemical or chemical method (Tsai et al., 2011; Bubnova et al., 2012), thus achieving the optimum TE properties through the control of charge carrier concentration. For instance, Park et al. reported PEDOT:PSS film with an optimized $P F$ of $112 \mu \mathrm{W} / \mathrm{K}^{2} \mathrm{~m}$ by treating with a mixture of DMSO and hydrazine (Park et al., 2014b). Also, Lee et al. employed a multistep process of ultrafiltration and dedoping by hydrazine to treat PEDOT:PSS and achieved a PF of $115.5 \mu \mathrm{W} / \mathrm{K}^{2} \mathrm{~m}$ (Lee et al., 2014a). Moreover, Park et al. reported an improved $P F$ of $1,270 \mu \mathrm{W} / \mathrm{K}^{2} \mathrm{~m}$ by controlling the electrochemical oxidation of PEDOT films (Park et al., 2013). Recently, Fan et al. treated PEDOT:PSS films with sulfuric acid and different concentrations of sodium hydroxide. They reported an improved $\sigma$ of $2,170 \mathrm{~S} / \mathrm{cm}$, an $S$ of $39.2 \mu \mathrm{V} / \mathrm{K}$, and hence a $P F$ of $334 \mu \mathrm{W} / \mathrm{K}^{2} \mathrm{~m}$ at room temperature (Fan et al., 2017). More recently, Fan et al. have demonstrated that 1ethyl-3-methylimidazolium dicyanamide (EMIM-DCA) treated PEDOT:PSS films had an ultrahigh $P F$ of $754 \mu \mathrm{W} / \mathrm{K}^{2} \mathrm{~m}$ and a ZT of 0.75 at room temperature (Fan et al., 2018). Moreover, Saxena 
et al. treated PEDOT:PSS film with EMIM-DCA in THF solution. They observed the simultaneous improvement of $\sigma$ and $S$, and a maximum $P F$ of $170 \mu \mathrm{W} / \mathrm{K}^{2} \mathrm{~m}$ had been obtained because of the binary nature of both ionic liquids and PEDOT:PSS (Saxena et al., 2019). Therefore, approaches that can only slightly lower the high $\sigma$ of formamide-treated PEDOT:PSS films while significantly improve the $S$ could be effective for improving the TE properties of PEDOT:PSS films.

In this work, we reported the enhancement of the $S$ and $Z T$ of PEDOT:PSS films with ionic liquids (ILs) treatment. The effect of anions associated with ILs on the TE properties of treated PEDOT:PSS films were also investigated, revealing that the type of anions played a somewhat role in affecting the TE properties. PEDOT:PSS films treated with 40 vol.\% IL, 1-butyl-3-methylimidazolium bis(trifluoromethanesulfonyl) amide (BMIM-TFSI), achieved a highest $P F$ of $239.2 \mu \mathrm{W} / \mathrm{K}^{2} \mathrm{~m}$, and it also demonstrated a very good environmental stability, indicating that our approach is potential for practical TE application in the future.

\section{EXPERIMENTAL SECTION}

\section{Materials}

PEDOT:PSS solution (Clevios PH 1000, PEDOT:PSS weight ratio $=1: 2.5$ and concentration by mass $=$ $1.3 \%$ ) was purchased from Heraeus. Formamide, sodium formaldehyde sulfoxylate (SFS), 1-butyl-3-methylimidazolium tetrafluoroborate (BMIM-BF4), 1-butyl-3-methylimidazolium trifluoromethanesulfonate (BMIM-OTf) or 1-butyl-3methylimidazolium bis(trifluoromethanesulfonyl) amide (BMIM-TFSI) were purchased from Sigma-Aldrich. All chemicals were used as received without further purification. The chemical structures of ionic liquids are shown in Scheme 1.

\section{Sample Preparation}

PEDOT:PSS solution was filtered using a $0.45 \mu \mathrm{m}$ poly (vinylene difluoride) PVDF syringe filter. The glass substrates were cleaned with deionized (DI) water, detergent, acetone, and isopropanol in an ultrasonic bath consecutively and dried with nitrogen gas. The glass substrate was subjected to ultraviolet (UV)-ozone surface treatment for $15 \mathrm{~min}$ before use.

\section{Pristine PEDOT:PSS Films}

PEDOT:PSS films were prepared by drop-casting a $300 \mu \mathrm{L}$ of PEDOT:PSS solution on the pre-cleaned glass substrate using a micropipette. The deposited sample was firstly dried in air at $50^{\circ} \mathrm{C}$ for 30 min to mainly drive off the $\mathrm{H}_{2} \mathrm{O}$ solvent and then kept at $80^{\circ} \mathrm{C}$ for another $10 \mathrm{~min}$ to further remove the residual solvent. Finally, the PEDOT:PSS films were annealed at $130^{\circ} \mathrm{C}$ for $10 \mathrm{~min}$ to ensure complete evaporation of the solvent. The final thickness of each dried PEDOT:PSS film was in the range of $8-10 \mu \mathrm{m}$.

\section{Formamide Post-treatment}

For the post-treatment process, $140 \mu \mathrm{L}$ formamide was first dropped onto the PEDOT:PSS films on a hot plate at $180^{\circ} \mathrm{C}$ and then the films were dried for about $10 \mathrm{~min}$ to remove the residual formamide. Afterward, the formamide treated PEDOT:PSS films were cooled to room temperature in air, and these films were rinsed with DI water and then dried again on a hot plate at $140^{\circ} \mathrm{C}$ for $5 \mathrm{~min}$. The detailed procedures for treatment can be found in our previous work (Kyaw et al., 2018).

\section{Sodium Formaldehyde Sulfoxylate (SFS) Post-treatment}

Next, treatment with a salt solution was performed by dropping $150 \mu \mathrm{L}$ aqueous SFS solution onto a formamide pretreated PEDOT:PSS film on a hot plate at $140^{\circ} \mathrm{C}$. The film became dry after $\sim 5 \mathrm{~min}$. The SFS treated PEDOT:PSS film was cooled to room temperature in air, rinsed with DI water for three times to wash away the salt, and then dried at $140^{\circ} \mathrm{C}$ again.

\section{IL Post-treatment}

The post-treatment with ILs was conducted at room temperature. Initially, three ILs (ILs) (BMIM-TFSI, BMIM-BF 4 , and BMIMOTf) with concentrations of i.e., 0, 20, 40, 60, 80, and 100 vol.\% ILs in methanol were prepared. Then pre-treated PEDOT:PSS films with formamide and SFS were further treated with ILs in methanol according to the following steps: $150 \mu \mathrm{l}$ IL in methanol was dropped onto PEDOT:PSS film at room temperature and left for $30 \mathrm{~min}$. Next, the samples were dried by the aid of blowing $\mathrm{N}_{2}$ gas at a pressure of $0.15 \mathrm{MPa}$ for another $30 \mathrm{~min}$ to remove the residual solvent of IL. Then the films were rinsed by dipping the films in DI water for three times and then finally annealed at an elevated temperature of $140^{\circ} \mathrm{C}$ under air to remove the residual solvent. These treated samples were cooled down to room temperature before TE property measurements. All treatments were conducted at their optimized treatment temperatures and conditions. Scheme 2 illustrates detailed PEDOT:PSS film preparation and post-treatment with formamide, SFS, and various ILs.

\section{Characterization}

The thicknesses of PEDOT:PSS films was measured before and after treatment using KLA-Tencor P-10 surface profiler with a detection limit of $100 \AA(10 \mathrm{~nm})$. The sheet resistance (Rs) of the films was determined by the four-point probe method (Laresta-GP MCP-T610 from Mitsubishi Chemical) at the room temperature. The Loresta-GP MCP-T610 meter includes standard accessories PSP probe (MCP-TP06P [4-pins, inter-pin distance $1.5 \mathrm{~mm}$, pin points $0.26 \mathrm{R}$, spring pressure $70 \mathrm{~g} /$ pin is intelligent)] and probe checker [RMH112 (MCPTP06P)]. The edges of the film were located at $10 \mathrm{~mm}$ from the measurement point. The $\sigma$ is the inverse of the resistivity $\rho_{H}$, which is calculated in terms of the equation: $\rho_{H}=R_{S} t$ where $R s$ and $t$ are resistance and thickness of the film, respectively (Krupka, 2013).

The $S$ was obtained with a homemade setup in a humiditycontrolled room with a relative humidity $(\mathrm{RH})$ of $55 \%$ (Figure S1). It consists of two stages (about $5 \mathrm{~mm}$ apart), one of which is integrated with a heater to generate a temperature gradient in the test sample. First, two Au electrodes with $20 \mathrm{~mm}$ long, $1 \mathrm{~mm}$ wide, and $2 \mathrm{~mm}$ apart were thermally evaporated on the film. To minimize the experimental error, PEDOT: PSS film 
<smiles>CCCCn1cc[n+](C)c1</smiles>

BMIM-TFSI<smiles>CCCCn1cc[n+](CP(F)F)c1</smiles>

BMIM-BF $_{4}$<smiles>CCCCn1cc[n+](C)c1</smiles>

BMIM-OTf<smiles>NC=O</smiles>

Formamide<smiles>[N+]OS(=O)CO</smiles><smiles>CC(C)c1sc([TlH])c2c1OCCO2</smiles>

PEDOT:PSS

SCHEME 1 | Chemical structures of BMIM-TFSI, BMIM-BF, BMIM-OTF, SFS, formamide, and PEDOT:PSS.

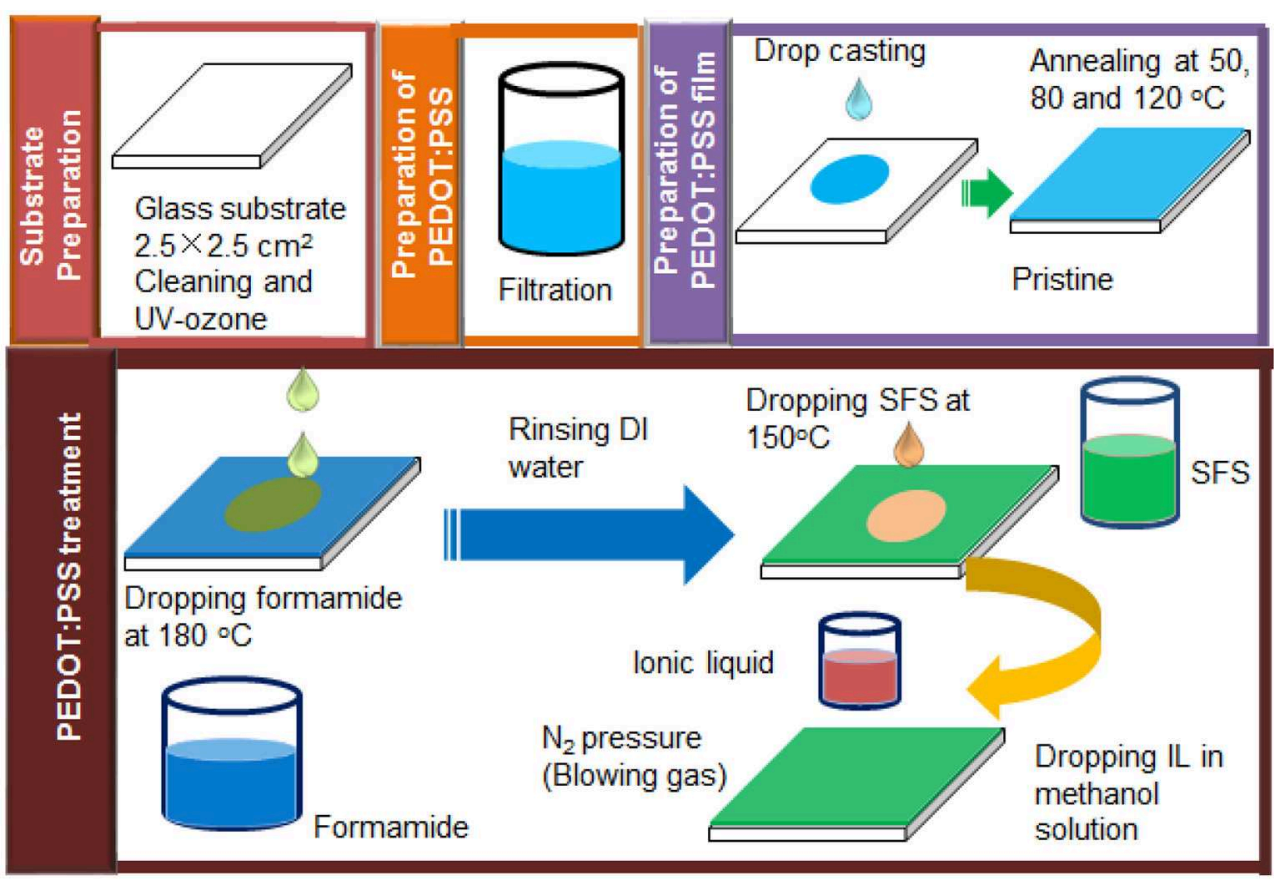

SCHEME 2 | Schematic of sample preparation for pristine and various IL-treated PEDOT:PSS films.

outside the area of electrodes was removed. The Au electrodes were connected to a Keithley 2,400 source meter through the probes to obtain the voltage difference $(\Delta V)$. Simultaneously, K-type thermocouples were connected to a data logger (Omron ZR-RX45) to collect the actual temperatures of the PEDOT:PSS film. The voltage probes and thermocouples were placed at the same temperature zone on each side so that the measured voltage corresponded to the actual thermal gradient between the two voltage probes. The measured thermovoltage was corrected by the thermovoltage of $\mathrm{Au}$ wire to obtain the absolute $S$ of the films. $S$ was estimated based on the slope of the linear relationship between thermoelectric voltage and the temperature difference of the two probes (i.e., $S=-\Delta V / \Delta T$ ). The Hall coefficient $R_{\mathrm{H}}$ was measured using a Hall-effect measurement system (Ecopia HMS-5000) with the van der Pauw method. First, the Ag electrodes were deposited onto the film through a shadow mask. The carrier concentration $n$ and mobility $\mu$ were calculated using the following equations: $n=1 /\left(\left|R_{H}\right| \times e\right)$ and $\mu=\left|R_{H}\right| / \rho_{H}$, where $e$ is electron charge. The $\kappa$ was calculated using the equation $\kappa=b^{2} / C p \rho_{m}$, where $\rho_{m}, b$, $C p$ are density, thermal effusivity, and specific heat capacity, respectively. The $b$ was measured with a Pulsed Light Heating Nano TR (NETZSCH) system with an ultrafast pulsed laser flash method using the front heating-front detection mode, which is 
designed for the thermal analysis of $30 \mathrm{~nm}-20 \mu \mathrm{m}$ thin films. The $C p$ was measured independently by differential scanning calorimeter (DSC) (Mettlier Toledo DSC1). The $\rho_{m}$ at room temperature was calculated from the mass and volume of the film. For all measurements, at least 10 samples for each measurement were prepared at the same conditions, and measurements were carried out at least five times for each sample to obtain the statistical results. X-ray diffraction (XRD) patterns of the films were obtained by a D8 Advance System (Bruker Corporation) equipped with a $\mathrm{Cu} K_{\alpha} \mathrm{X}$-ray source, $\lambda=0.15406 \mathrm{~nm}$. The Raman spectroscopy measurements were conducted on a Raman microscope (Renishaw) with a laser wavelength of $785.5 \mathrm{~nm}$, a laser beam spot size of $200 \mu \mathrm{m}$ and an accumulation time of $30 \mathrm{~s}$. The absorption spectra measurement was performed on a UV-Vis-NIR spectrophotometer (Shimadzu, UV-3600). The films were spin-coated on quartz substrates. X-ray photoelectron spectroscopy (XPS) of the films were obtained by the Theta Probe Angle-Resolved X-ray Photoelectron Spectrometer (ARXPS) System (Thermo Scientific) using monochromated, microfocused $\mathrm{Al} K_{\alpha} \mathrm{X}$-ray photons $(h v=1486.6 \mathrm{eV}$ ) at a base pressure of $1 \times 10^{-9}$ Torr and a step size of $0.1 \mathrm{eV}$. The curve fitting was carried out using the Avantage software. Atomic force microscopy (AFM) images were taken on a Bruker Dimension Icon AFM using the tapping mode. Ultraviolet photoelectron spectroscopy (UPS) measurement of the films was obtained by using the He I photon $(21.22 \mathrm{eV})$ radiation line from a discharge lamp, with an experimental resolution of $0.15 \mathrm{eV}$. All UPS measurements of the onset of photoemission for determining the $\Phi$ were performed using standard procedures with a $-4.5 \mathrm{~V}$ bias applied to the sample. The films were prepared by drop-casting a PEDOT:PSS solution onto a pre-cleaned silicon substrate.

\section{Stability Study of PEDOT:PSS Films}

PEDOT:PSS films were placed in a humidity controlled chamber and their TE performance was measured at varied temperature and humidity conditions in order to study the effect of humidity on TE properties of PEDOT:PSS films (Kim et al., 2016). In this study, the stability study was conducted using a constant climate chamber (Memmert HPP 110) in the temperature range from $+0^{\circ} \mathrm{C}$ to $+70^{\circ} \mathrm{C}$, as well as the active humidification and dehumidification from 10 to $90 \% \mathrm{RH}$.

\section{RESULTS AND DISCUSSION}

\section{Film Post-treatment and TE Properties}

Three different ILs, 1-butyl-3-methylimidazolium bis(trifluoromethanesulfonyl) amide (BMIM-TFSI), 1butyl-3-methylimidazolium tetrafluoroborate (BMIM-BF 4$)$ or 1-butyl-3-methylimidazolium trifluoromethanesulfonate (BMIM-OTf) in methanol were used for this study. ILs are composed of positively and negatively charged species (i.e., binary nature). In these three ILs, the cation is always BMIM, and only the anion is different. All post-treatment methods are summarized in Table 1.

Figures 1A,C,E show the $S, \sigma$, and PF of the PEDOT:PSS films treated by sequential formamide and ILs with various concentrations. The PEDOT:PSS films were pre-treated with
TABLE 1 | Post-treatments methods of PEDOT:PSS films.

\begin{tabular}{|c|c|c|c|}
\hline $\begin{array}{l}\text { Post- } \\
\text { treatment } \\
\text { Series }\end{array}$ & $\begin{array}{l}\text { Post- } \\
\text { treatment } \\
\text { methods }\end{array}$ & $\begin{array}{l}\text { Reagents } \\
\text { used }\end{array}$ & Procedures \\
\hline \multirow[t]{2}{*}{1} & $\begin{array}{l}\text { F- } \\
\text { PEDOT:PSS }\end{array}$ & Formamide & $\begin{array}{l}\text { Drop } 140 \mu \mathrm{L} \text { formamide onto the } \\
\text { PEDOT:PSS films at } 180^{\circ} \mathrm{C} \text { and } \\
\text { dry for about } 10 \mathrm{~min} \text {. Then rinse } \\
\text { with DI water and dry again on a } \\
\text { hot plate at } 140^{\circ} \mathrm{C} \text { for } 5 \mathrm{~min} \text {. }\end{array}$ \\
\hline & $\begin{array}{l}\text { ILs-F- } \\
\text { PEDOT:PSS }\end{array}$ & $\begin{array}{l}\text { Formamide + } \\
\text { Ionic liquid }\end{array}$ & $\begin{array}{l}\text { The three ILs (BMIM-TFSI, } \\
\text { BMIM-BF4, and BMIM-OTf) with } \\
0,20,40,60,80 \text {, and } 100 \text { vol. } \% \\
\text { ILs in methanol were prepared } \\
\text { and was dropped onto } \\
\text { F-PEDOT:PSS film at room } \\
\text { temperature and left for } 30 \text { min } \\
\text { and finally dry blowing } \mathrm{N}_{2} \text { gas. } \\
\text { Then rinse with DI water and dry } \\
\text { again on a hot plate at } 140^{\circ} \mathrm{C} \text { for } \\
5 \text { min. }\end{array}$ \\
\hline \multirow[t]{3}{*}{2} & $\begin{array}{l}\text { F- } \\
\text { PEDOT:PSS }\end{array}$ & Formamide & $\begin{array}{l}\text { Drop } 140 \mu \mathrm{L} \text { formamide onto the } \\
\text { PEDOT:PSS films at } 180^{\circ} \mathrm{C} \text { and } \\
\text { dry for about } 10 \mathrm{~min} \text {. Then rinse } \\
\text { with DI water and dry again on a } \\
\text { hot plate at } 140^{\circ} \mathrm{C} \text { for } 5 \mathrm{~min} \text {. }\end{array}$ \\
\hline & $\begin{array}{l}\text { SFS-F- } \\
\text { PEDOT:PSS }\end{array}$ & $\begin{array}{l}\text { Formamide }+ \\
\text { Sodium } \\
\text { formaldehyde } \\
\text { sulfoxylate }\end{array}$ & $\begin{array}{l}\text { Drop } 150 \mu \mathrm{L} \text { aqueous SFS } \\
\text { solution onto F-PEDOT:PSS film } \\
\text { at } 140^{\circ} \mathrm{C} \text { and dry for about } \\
5 \mathrm{~min} \text {. Then rinse with DI water } \\
\text { and dry again on a hot plate at } \\
140^{\circ} \mathrm{C} \text { for } 5 \text { min. }\end{array}$ \\
\hline & $\begin{array}{l}\text { ILS-SFS-F- } \\
\text { PEDOT:PSS }\end{array}$ & $\begin{array}{l}\text { Formamide }+ \\
\text { Sodium } \\
\text { formaldehyde } \\
\text { sulfoxylate }+ \\
\text { lonic liquid }\end{array}$ & $\begin{array}{l}\text { The three ILs (BMIM-TFSI, } \\
\text { BMIM-BF4, and BMIM-OTf) with } \\
\text { O, 20, } 40,60,80 \text {, and } 100 \text { vol. } \% \\
\text { ILs in methanol were prepared } \\
\text { and was dropped onto } \\
\text { SFS-F-PEDOT:PSS film at room } \\
\text { temperature and left for } 30 \text { min } \\
\text { and finally dry blowing } \mathrm{N}_{2} \text { gas. } \\
\text { Then rinse with DI water and dry } \\
\text { again on a hot plate at } 140^{\circ} \mathrm{C} \text { for } \\
5 \text { min. }\end{array}$ \\
\hline
\end{tabular}

formamide, and then ILs in methanol were dispersed onto the PEDOT:PSS films (first series post-treatment: IL-F-PEDOT:PSS). Introduction of IL treatment reduced the $\sigma$, while noticeably improved the $S$ of IL-F-PEDOT:PSS films. It is worth noting that the $\sigma$ and $S$ of IL-F-PEDOT:PSS films almost remain unchanged when the concentration of ILs increases from 20 to $80 \%$, suggesting that they are less affected by the concentration of the ILs. Treatment with 20-100 vol.\% BMIM-TFSI (i.e., BMIMTFSI-F-PEDOT:PSS) led to an enhancement of the $S$ from $\sim 14.9$ to $\sim 28.1 \mu \mathrm{V} / \mathrm{K}$ and a significant reduction in the $\sigma$ from $\sim 2,873$ to $\sim 1,678 \mathrm{~S} / \mathrm{cm}$. Overall, in comparison to the $P F$ of around $65 \mu \mathrm{W} / \mathrm{K}^{2} \mathrm{~m}$ without IL treatment, the optimal $P F$ reaches $137.8 \mu \mathrm{W} / \mathrm{K}^{2} \mathrm{~m}$ when $40 \mathrm{vol} \%$ BMIM-TFSI solution is used. Interestingly, the type of anions plays a certain role in improving $S$. TFSI is the most efficient to enhance the magnitude of the $S$, followed by OTf and $\mathrm{BF}_{4}$. The possible rationale is because of the binary nature of both PEDOT:PSS and ILs, which consist of 

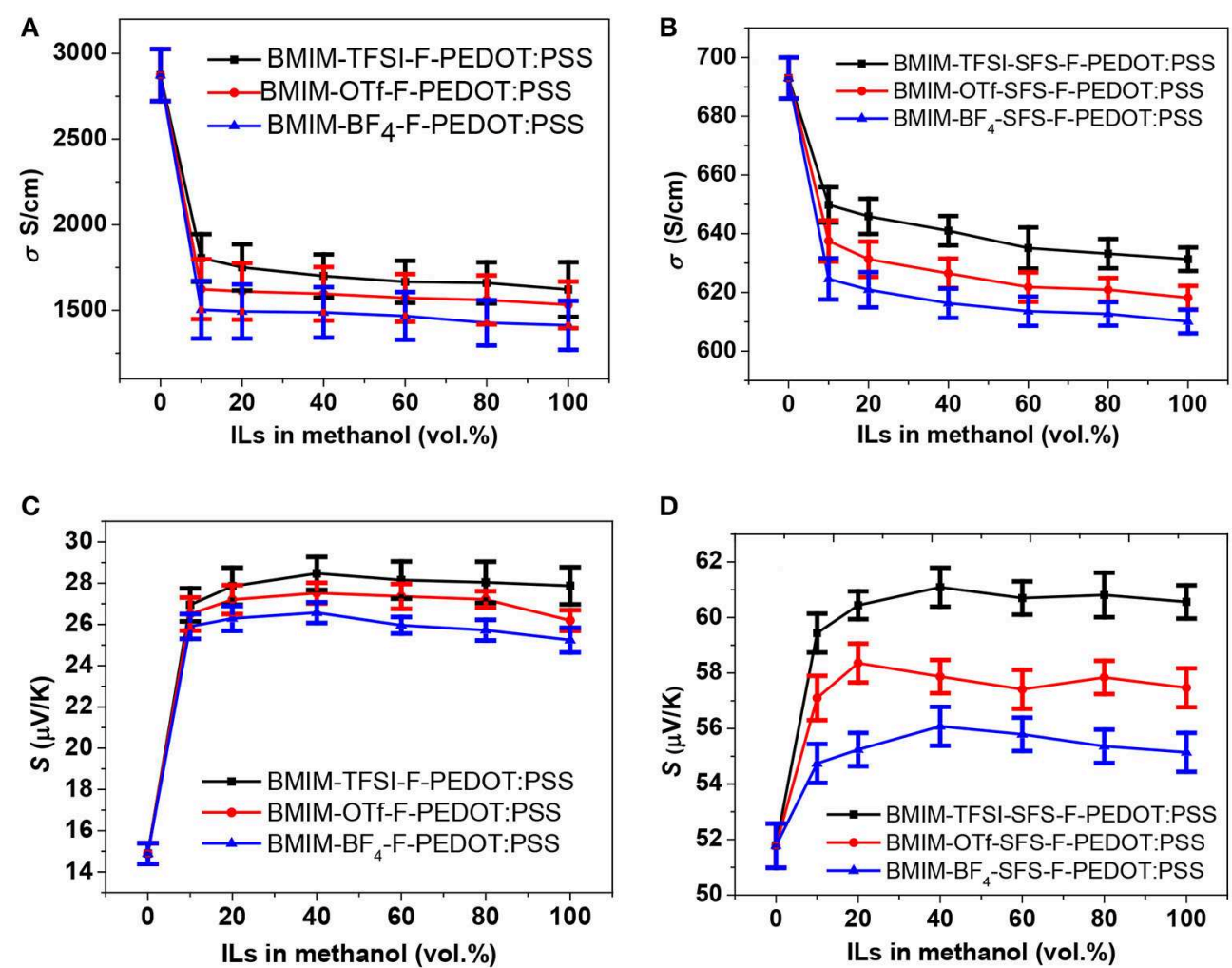

D
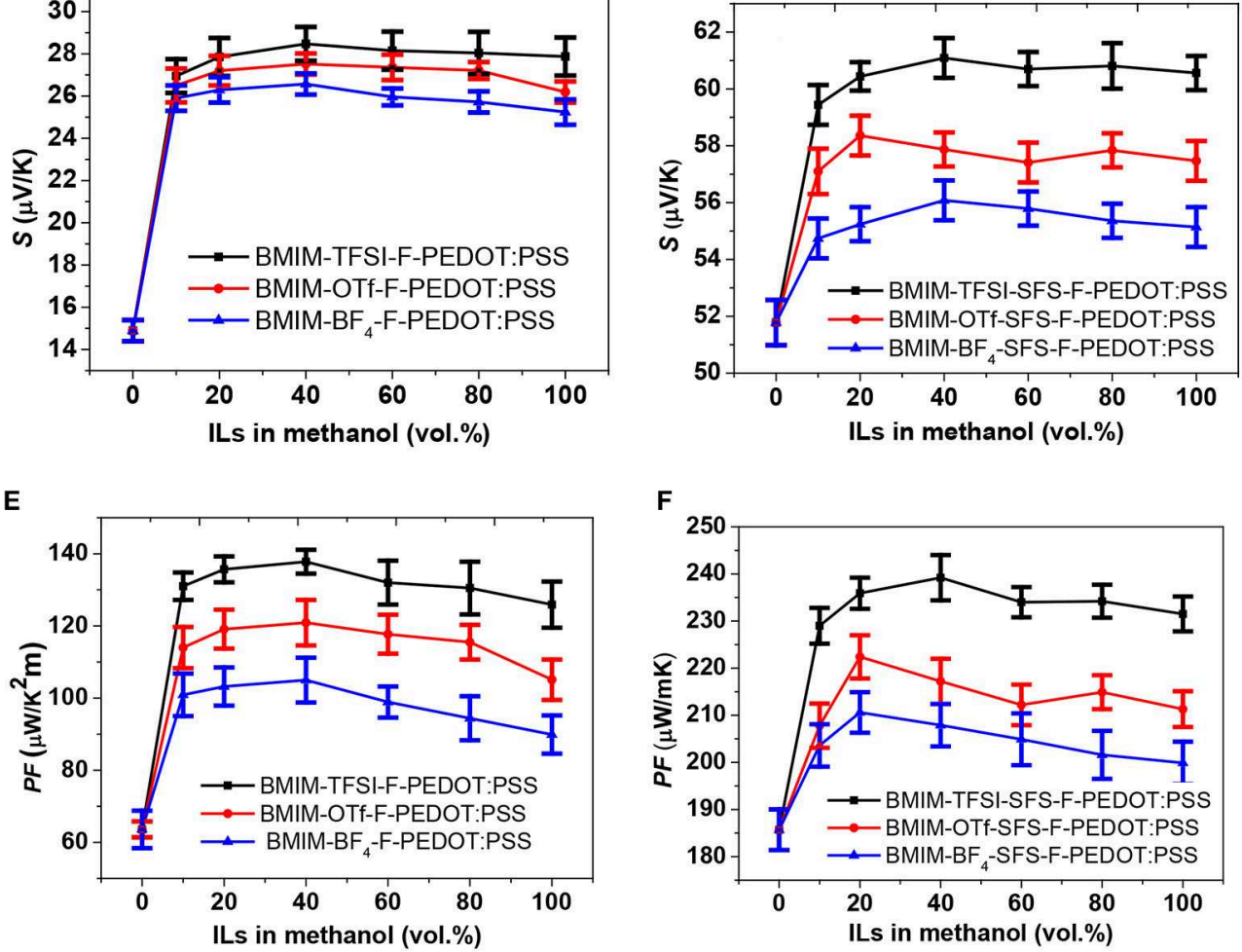

FIGURE 1 | TE properties of PEDOT:PSS films vs. vol.\% ILs. ILs-F-PEDOT:PSS films: (A) $\sigma$, (C) S, and (E) PF; ILs-SFS-F-PEDOT:PSS films: (B) $\sigma$, (D) S, and (F) PF.

positively and negatively charged species. These charged species in ILs, for example, negatively charged TFSI and positively charged BMIM, will interact with correspondingly positively charged polymer PEDOT and negatively charged PSS chains via electrostatic interactions.

In the second series post-treatment, SFS was introduced to treat PEDOT:PSS before applying ILs treatment. Based on our previous findings (Yemata et al., unpublished), subsequent treatment of F-PEDOT:PSS with SFS can reduce the doping level, and as a result, improve the PF of PEDOT:PSS films. The $\sigma, S$, and $P F$ of the ILs-SFS-F-PEDOT:PSS films with different concentrations of ILs are summarized in Figures 1B,D,F. The $\sigma$ of ILs-SFS-F-PEDOT:PSS film was only slightly lower than that of the SFS-F-PEDOT:PSS films (631-649 vs. $693 \mathrm{~S} / \mathrm{cm}$ ), while the $\mathrm{S}$ became higher. BMIM-TFSI-SFS-F-PEDOT:PSS films exhibited an improved $S$ of $\sim 60.8 \mu \mathrm{V} / \mathrm{K}$ compared to SFS-F-PEDOT:PSS $(51.8 \mu \mathrm{V} / \mathrm{K})$, while the $\sigma(\sim 631 \mathrm{~S} / \mathrm{cm})$ decreased slightly. Consequently, the $P F$ of BMIM-TFSI-SFS-FPEDOT:PSS reached $239 \mu \mathrm{W} / \mathrm{K}^{2} \mathrm{~m}$, about $29 \%$ enhancement over that of F-SFS-PEDOT:PSS. Similar to the first series post-treatment, the $P F$ was nearly independent on the IL concentration, resulting in an average $P F$ of $235 \mu \mathrm{W} / \mathrm{K}^{2} \mathrm{~m}$ for BMIM-TFSI-SFS-F-PEDOT:PSS films. These values are among the highest PFs reported in the literature (Table S2) (Bubnova et al., 2011; Lee et al., 2014a; Park et al., 2014b; Wang et al., 2015; Yi et al., 2015; Fan et al., 2017). This enhancement in the $P F$ 

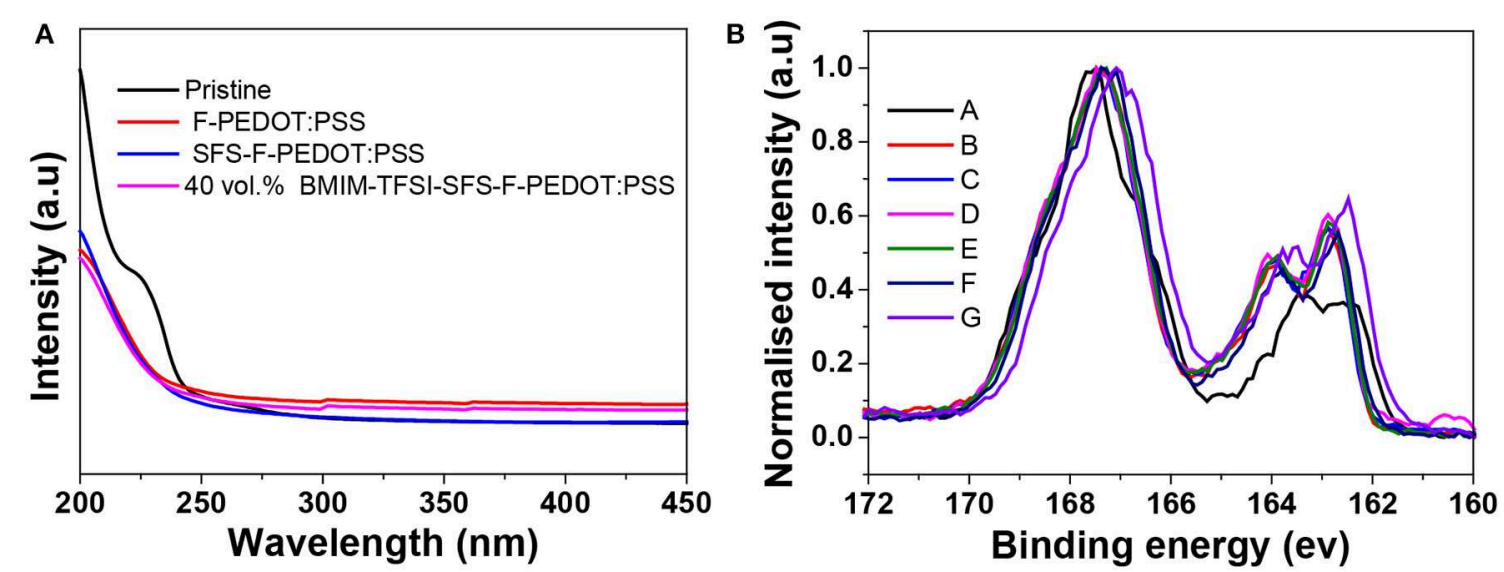

FIGURE 2 | (A) UV-vis absorption spectra of the pristine, F-PEDOT:PSS, SFS-F-PEDOT:PSS and BMIM-TFSI-SFS-F-PEDOT:PSS films. (B) S 2p core-level spectra of the pristine, F-PEDOT:PSS, and BMIM-TFSI-SFS-F-PEDOT:PSS films treated with various vol.\% BMIM-TFSI in methanol. Pristine (A), F-PEDOT:PSS (B), SFS-F-PEDOT:PSS (C), 40 vol.\% BMIM-TFSI-SFS-F-PEDOT:PSS (D), 60 vol.\% BMIM-TFSI-SFS-F-PEDOT:PSS (E), 80 vol.\% BMIM-TFSI-SFS-F-PEDOT:PSS (F), and 100 vol.\% BMIM-TFSI-SFS-F-PEDOT:PSS (G).

is mainly due to the interaction of $\mathrm{PSS}^{-}$groups with $\mathrm{BMIM}^{+}$, which tunes $n$ in the PEDOT domains and thus significantly enlarges the $S$ but does not compromise the $\sigma$ too much. Besides, control samples were also prepared by treating the F-PEDOT:PSS and SFS-F-PEDOT:PSS films with pure methanol (i.e., $\mathrm{MeOH}-$ F-PEDOT:PSS and MeOH-SFS-F-PEDOT:PSS) to verify that the observed changes are not related to interactions of the solvent with the PEDOT:PSS film. MeOH-F-PEDOT:PSS and $\mathrm{MeOH}-$ SFS-F-PEDOT:PSS control films showed PFs of 92.5 and 189 $\mu \mathrm{W} / \mathrm{K}^{2} \mathrm{~m}$, respectively. These values are smaller than those of the IL-F-PEDOT:PSS and IL-SFS-F-PEDOT:PSS films. Therefore, ILs in methanol has a more significant influence on the $S$ and $\sigma$ of PEDOT:PSS films than pure methanol.

\section{Mechanism of TE Properties Enhancement of ILs Treated Films}

Figure 2A shows the UV absorption spectra of the pristine, FPEDOT:PSS and SFS-F- PEDOT:PSS and BMIM-TFSI-SFS-FPEDOT:PSS films. The absorption band located at $225 \mathrm{~nm}$ was assigned to the PSS. Generally, the decreased intensity of the absorption band at $225 \mathrm{~nm}$ of PEDOT:PSS films shows the loss of PSSH from the PEDOT:PSS films. Compared with the spectrum of F-PEDOT:PSS that showed remarkable reduction in intensity at $225 \mathrm{~nm}$, both spectra of SFS-F-PEDOT:PSS and BMIM-TFSISFS-F-PEDOT:PSS were almost the same, indicating that, similar to other treatments (Xia et al., 2012), formamide-treatment effectively removed $\mathrm{PSSH}$, resulting in an increase in the $\sigma$ compared to the pristine film.

The $\mathrm{S}_{2} \mathrm{p}$ X-ray photoemission spectroscopy (XPS) was employed to study the influence of IL treatment (Figure 2B). The doublet XPS bands with binding energies between 166 and $172 \mathrm{eV}$ were assigned to the $\mathrm{S}_{2} \mathrm{p}$ band of the sulfur atoms in PSS, whereas XPS peaks with binding energies between 162 and $166 \mathrm{eV}$ were assigned to the $S_{2} p$ band of the sulfur atoms of PEDOT (Crispin et al., 2003; Kim et al., 2011). It can be seen from Figure $2 \mathrm{~B}$ that the $\mathrm{S}_{2} \mathrm{p}$ intensity of PEDOT relative to PSS increases due to the removal of PSS after BMIM-TFSI treatment (Xia and Ouyang, 2009). The ratios of the PSS peak to PEDOT peak dropped from 2.5 for the pristine film to 1.02 for the SFSF-PEDOT:PSS film, indicating that a substantial amount of PSS was removed in the treated films. The removal of PSS was also verified by the reduction in the film thickness. The thickness of the pristine film was $6 \mu \mathrm{m}$. In contrast, the thickness of ILSFS-F-PEDOT:PSS, F-PEDOT:PSS and SFS-F-PEDOT:PSS film was reduced significantly to $2.3,2.5$, and $2.1 \mu \mathrm{m}$, respectively. Also, the S2p bands in the PEDOT increased and shifted to a higher binding energy (163.5 ev vs. $164.2 \mathrm{ev)}$ after IL treatment, indicating the decrease in the doping level after treatment, and the lower doping level in CPs invariably led to a reduced $\sigma$. The dedoping with IL slightly affected the $\sigma$ while significantly improve the $S$. The PF of PEDOT:PSS film attained its optimum value at a specific oxidation state as the $S$ increased and the $\sigma$ tends to decrease at lower oxidation levels, which is consistent with the previous report (Khan et al., 2000).

The oxidation level of PEDOT:PSS thin films treated with the chemical dedoping agent was determined with UV-Vis-NIR absorption spectroscopy (Figure 3A). PEDOT exists in a form of neutral, polaron (a radical cation charge carrier) and bipolaron (a di-cation charge carrier) state (Figure 3B). The pristine and F-PEDOT:PSS films show a broad absorption band covering the beginning of the infrared region domain, and this band is attributed to bipolaron (PEDOT ${ }^{2+}$ ). After binary dedoping (IL-F-PEDOT:PSS \& IL-SFS-F-PEDOT:PSS), the oxidation level changes and the main peaks shifted to $900 \mathrm{~nm}$ for polaron $\left(\mathrm{PEDOT}^{+}\right)$and to $600 \mathrm{~nm}$ for neutral (PEDOT) redox states (Chung et al., 1984; Garreau et al., 2001; Im and Gleason, 2007; Bubnova et al., 2011). Nevertheless, this further binary dedoping with ILs steps leads to the formation of neutral states of PEDOT chains at a high intensity (i.e., the absorption intensity is higher for IL-SFS-F-PEDOT:PSS films than that for SFS-F-PEDOT:PSS 

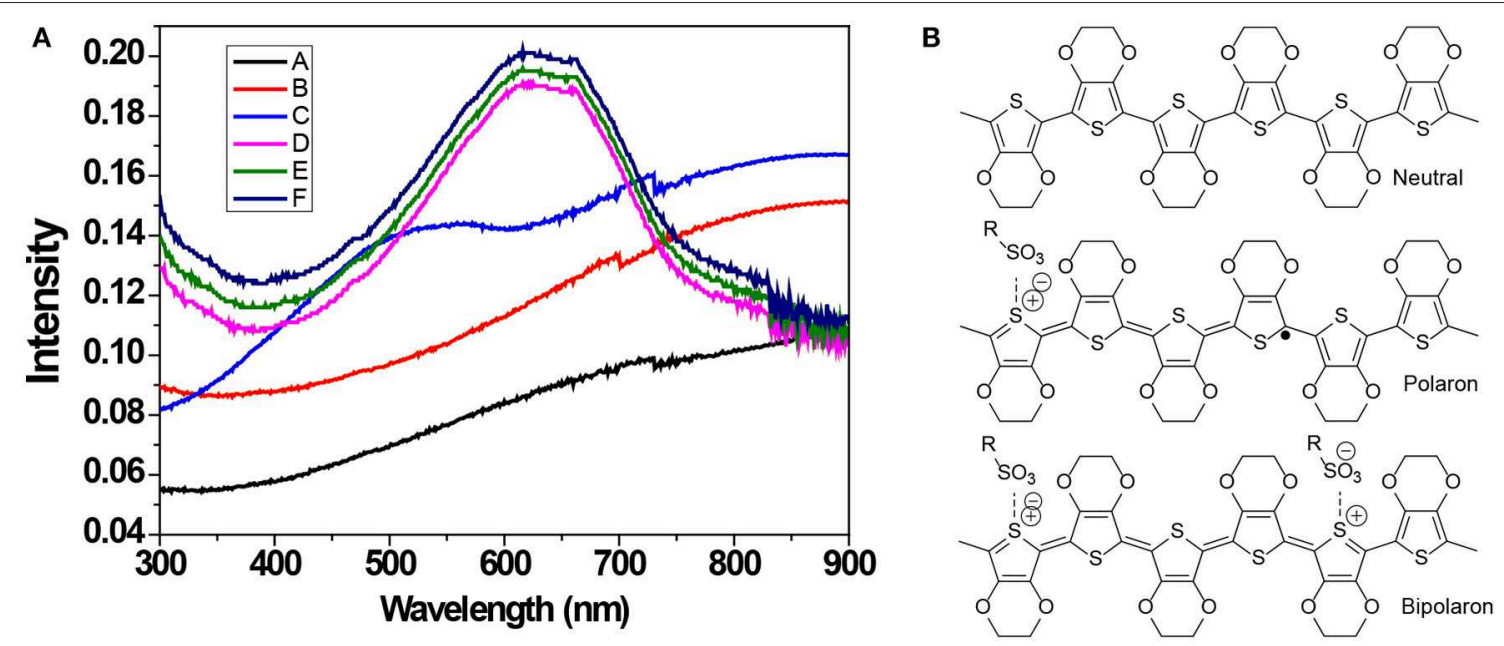

FIGURE 3 | (A) UV-Vis-NIR absorption spectra of pristine, F-PEDOT:PSS, and BMIM-TFSI-SFS-F-PEDOT:PSS films treated with various vol.\% of BMIM-TFSI in methanol. Pristine (A), F-PEDOT:PSS (B), SFS-F-PEDOT:PSS (C), 40 vol.\% BMIM-TFSI-SFS-F-PEDOT:PSS (D), 60 vol.\% BMIM-TFSI-SFS-F-PEDOT:PSS (E), and 80 vol.\% BMIM-TFSI-SFS-F-PEDOT:PSS (F). (B) A schematic Illustration of the transition of PEDOT chains from bipolaron (a di-cation charge carrier) to polaron (a radical cation charge carrier) and neutral chain during dedoping with IL.

films). This indicates that the PEDOT chains in the neutral state can remain in the neutral states upon binary dedoping but with a higher intensity, resulting in further enhancement in the $S$ due to a lower $n$.

Raman spectroscopy was used to further investigate the conformational changes in polymers and to study the change in the doping level of PEDOT:PSS films. Figure 4A displays the Raman spectra of the untreated and BMIM-TFSI-SFS-FPEDOT:PSS treated films. The peaks at 987, 1,130, and 1,257 $\mathrm{cm}^{-1}$ came from the deformation of oxyethylene ring, the PSS component, and the vibrational mode of $\mathrm{C} \alpha-\mathrm{C} \alpha$ ' symmetric interring stretching, respectively (Garreau et al., 1999, 2001; Han et al., 2011; Farah et al., 2012). Also, the peak around 1,509 $\mathrm{cm}^{-1}$ was originated from the asymmetrical vibration of $\mathrm{C}_{\alpha}=$ $\mathrm{C}_{\beta}$ in PEDOT and the peak around $1,369 \mathrm{~cm}^{-1}$ was assigned to the symmetric C $\beta-C \beta$ stretching (Garreau et al., 1999). The structure of the pristine PEDOT is made up of the benzoid and quinoid forms in which the conjugated benzoid structure owns a localized $\pi$-electron largely unaffected by external stimuli, while the quinoid form of PEDOT holds a delocalized state of $\pi$ electrons which can be affected by solvent treatment (Ouyang et al., 2005). In the electrically active and oxidized state, there are positive charges on the PEDOT polymer backbone which are balanced with an anion, either a small molecular anion or a macromolecular anion such as the PSS ${ }^{-}$(Ouyang et al., 2005). The vibrational bands at $1424 \mathrm{~cm}^{-1}$ can be ascribed to the stretching vibration on the $\mathrm{C} \alpha=\mathrm{C} \beta$ of the five-member ring of the pristine PEDOT films. These vibrational bands were shifted to around 1,417 $\mathrm{cm}^{-1}$ for IL-SFS-F-PEDOT:PSS films (Figure 4B), suggesting a change from a predominately coil conformation (benzoid structure) to a mixed linear-coil conformation (quinoid structure) in the PEDOT chain (Garreau et al., 1999; Łapkowski and Pron, 2000), resulting in a quinoid dominant structure. The PSS chains are connected to the PEDOT chains through
Coulombic interactions and have a coiled structure (core-shell) because of the repulsion among the long PSS chains (Lang et al., 2009a). This IL treatment could weaken the ionic interaction between the PEDOT and the PSS, resulting in phase separation between the PSS and the PEDOT and a linear conformation of the PEDOT chains. The same observations were reported on Raman analysis of EG treated PEDOT:PSS films (Ouyang et al., 2004). The partial removal of PSS is manifested by the reduction in the intensity of the Raman fingerprints of treated films compared to pristine films. Moreover, the peak at 1,424 $\mathrm{cm}^{-1}$ for the pristine shifted to $1,417 \mathrm{~cm}^{-1}$ upon IL treatment (Figure 4B), indicating that the doping level changed from bipolaron in the pristine to a neutral state in the IL-treated films as evident by UV-vis-NIR spectra and XPS. This Raman spectra along with UV-vis-NIR spectra and XPS indicate that the oxidation level changes from bipolaron to neutral upon dedoping lead to the slight decrease in the $\sigma$ and the significant increase in the $S$ due to the decrease in the $n$ (Luo et al., 2013).

The surface of treated films is highly non-uniform and with enhanced particle size, which leads to a more readily charge transport and thus an improved $\sigma$ compared to the pristine film. While the untreated film does not show any apparent grains (Figures S2a,d), implying that the PSS chains are well-intermixed with the PEDOT chains and the PSS-rich domains mostly cover the film. The strong phase separation between the PSS-rich shell and the PEDOT-rich core besides the depletion of PSS chain were found in the treated films resulting in the interconnected large grains of PEDOT (Figure S2 and Figure 5) (Na et al., 2009; Luo et al., 2013, 2014). After dedoping with ILs the interconnection of the PEDOT-rich grains was enhanced, resulting in an enhanced $\sigma$ compared to the pristine film. This could partially address why ILs treatment improves $P F$ with a slight degradation of the $\sigma$. 
A
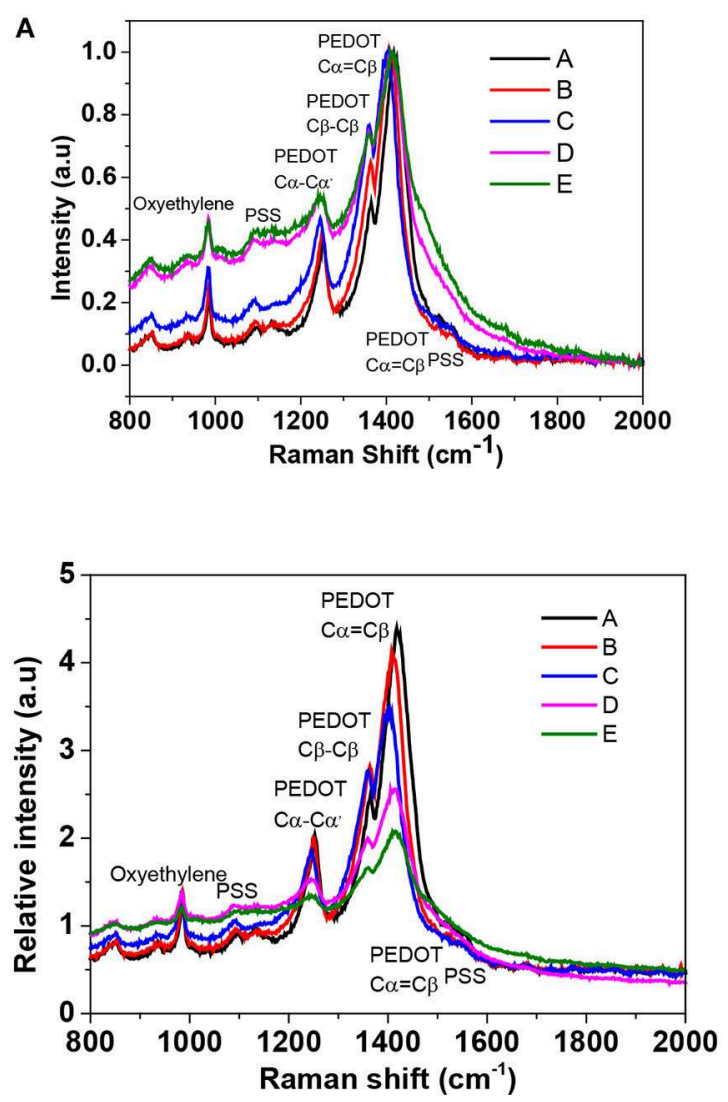

B
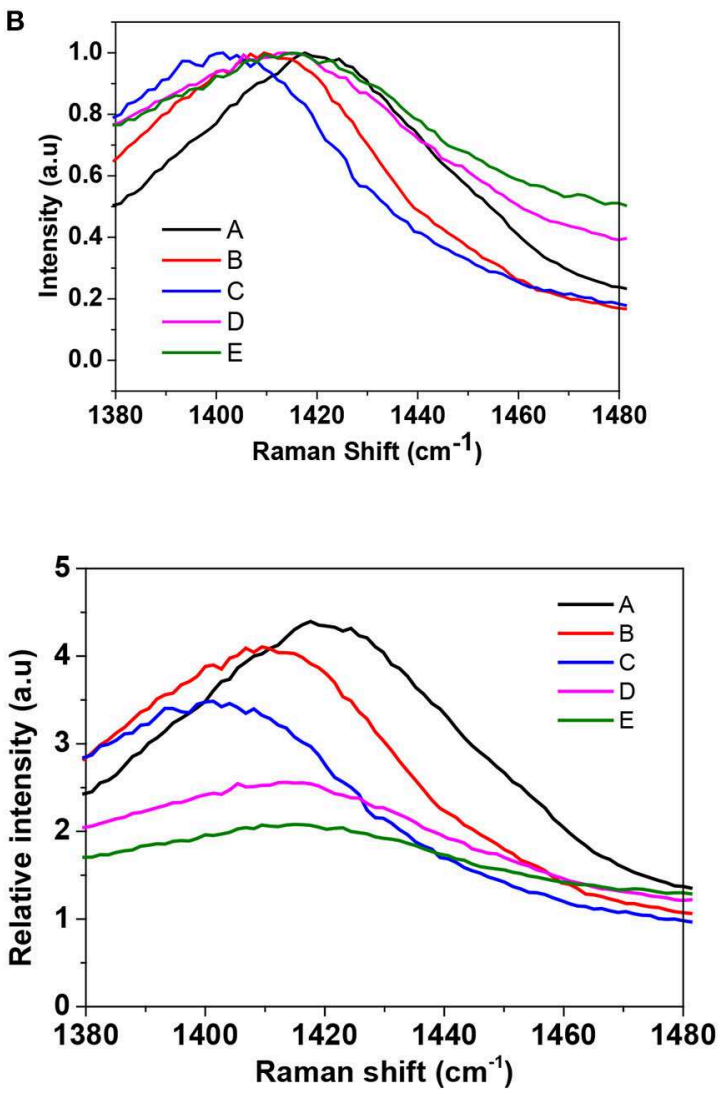

FIGURE 4 | (A) Raman spectra of pristine PEDOT:PSS film and BMIM-TFSI-SFS-F-PEDOT:PSS films treated with different vol.\% of BMIM-TFSI in methanol. Pristine (A), SFS-F-PEDOT:PSS (B), 40 Vol.\% BMIM-TFSI-SFS-F-PEDOT:PSS (C), 60 Vol.\% BMIM-TFSI-SFS-F-PEDOT:PSS (D), and 80 Vol.\% BMIM-TFSI-SFS-F-PEDOT:PSS (E). (B) Zoom in spectra in the wavelength range of 1,380 to 1,480 $\mathrm{cm}^{-1}$.

Hall measurements were carried out to measure the $n$ and the $\mu$ in order to further confirm the conformational change of the PEDOT chain and the phase segregation of the PSSH due to the binary dedoping with the IL. The results showed that all the prepared films were hole-type carrier dominated. In the $p$ type semiconductors, the $\sigma$ is given by the relationship: $\sigma=e n \mu$, where $n, e$, and $\mu$ is the charge carrier concentration, electron charge, and charge carrier mobility, respectively (Hiroshige et al., 2007). The $\sigma$ of the pristine and treated PEDOT:PSS films (as shown in Table 2) is in the same order of magnitude with the measured value (Table S1), demonstrating the reliability of the current measurements. The slightly decreased $\sigma$ of the ILs dedoped PEDOT:PSS film was mainly caused by the one-orderof-magnitude reduction in the $n$ as the $\mu$ varied slightly. This may be ascribed to the interaction between ILs molecules and the PSS monomers, and thus inhibit the carrier supply from the PSS. Generally, the $S$ effectively varies with the slight doping or dedoping concentration as manifested in the current work that the dedoping effect of ILs contributes to the significant enhancement in the $S$.

In addition to TE performance of the films, the ILs treatment on the PEDOT:PSS films may also affect other properties of PEDOT:PSS films relevant to the device operation, such as the work function $(\Phi)$. Ultraviolet photoelectron spectroscopy (UPS) is a key technique to determine the $\Phi$ of surfaces by measuring the secondary-electron cut-off $(\mathrm{Ec})$. The influence of the $\Phi$ and the valence band on the pristine and treated PEDOT:PSS films was determined using UPS measurements (Figure 6). The $\Phi$ could be obtained from the equation, $\Phi=$ $h v-E c$, based on the UPS measurements where the spectral width secondary-electron cut-off $(E c)$ is obtained from the energy gap between the inelastic secondary electron emission cutoff and the Fermi edge and $h v$ is the photon energy of the UPS light source (Janardhanam et al., 2015; Kim et al., 2018). We found that IL treatment caused a reduction in the $\Phi$ from 4.7 to $4.4 \mathrm{eV}$ (Figure 6). For PEDOT:PSS thin films, a range of the $\Phi$ from 4.7 to $5.4 \mathrm{eV}$ has been reported (Scott et al., 1999; Greczynski et al., 2001; Mäkinen et al., 2001; Havare et al., 2012) which is similar to the result shown in Figure 6. The spread in the $\Phi$ values is assumed to be related to differences in the top layer, which may contain an excess of the PSS (Jönsson et al., 2003; Huang et al., 2005; Snaith et al., 2005; Crispin et al., 2006). The PSS-rich top layer may be modified by the addition of high-boiling solvents (Huang et al., 2005; Snaith et al., 2005; Hwang et al., 2006) and other processing conditions (Koch et al., 2007). 


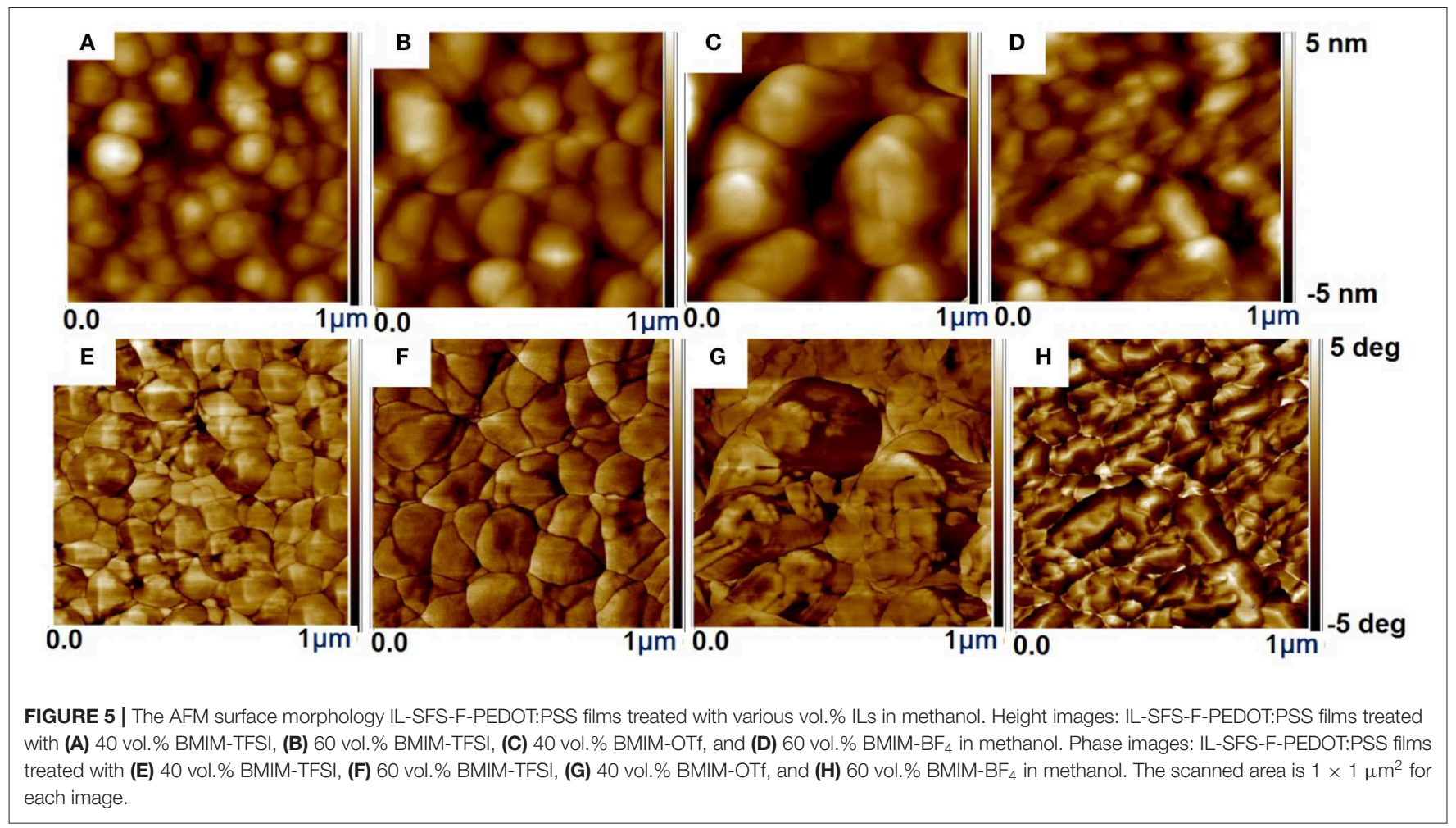

TABLE 2 | Calculated $\sigma$ and experimentally determined $n$ and $\mu$ of the sequential formamide (three times) and $100 \mathrm{mM}$ SFS pre-treated PEDOT:PSS films at various vol.\% BMIM-TFSI in methanol by the aid of blowing $\mathrm{N}_{2}$ gas.

\begin{tabular}{lccc}
\hline $\begin{array}{l}\text { Treatment methods } \\
\text { (vol.\% BMIM-TFSI } \\
\text { in methanol) }\end{array}$ & $\boldsymbol{\mu}\left(\mathbf{c m}^{2} \mathbf{N s}\right)$ & $\boldsymbol{n}\left(\mathbf{c m}^{-3}\right)$ & $\boldsymbol{\sigma} \mathbf{( S / c m )}$ \\
\hline Untreated & & & \\
F-PEDOT:PSS & $0.42 \pm 0.03$ & $4.1 \pm 0.3 \times 1018$ & $0.28 \pm 0.1$ \\
0 & $1.08 \pm 0.1$ & $1.56 \pm 0.1 \times 1022$ & $2693.7 \pm 201.8$ \\
20 & $0.58 \pm 0.04$ & $5.32 \pm 0.5 \times 1021$ & $493.72 \pm 41$ \\
40 & $0.51 \pm 0.05$ & $5.11 \pm 0.3 \times 1021$ & $416.97 \pm 37$ \\
60 & $0.48 \pm 0.04$ & $5.01 \pm 0.7 \times 1021$ & $384.77 \pm 31$ \\
80 & $0.43 \pm 0.04$ & $4.87 \pm 0.63 \times 1021$ & $335.05 \pm 29$ \\
100 & $0.41 \pm 0.05$ & $4.65 \pm 0.45 \times 1021$ & $305.04 \pm 21$ \\
\hline
\end{tabular}

The $S$ gives the energetic difference between the transport level $(E \mu)$ and the Fermi level $\left(E_{F}\right)$ by its value and the transport type ( $p$ or $n$ by its sign). The theoretical result indicates, the $S$ generally depends on the Fermi level as expressed (Fritzsche, 1971; Nollau et al., 2000):

$$
S(T)=\frac{1}{e T} \frac{\int\left[E_{F}(T)-E\right] \delta \sigma(E) d E}{\int \delta \sigma(E) d E}
$$

where $\delta \sigma(E)$ is the differential conductivity at energy $E, E_{F}$ is the Fermi level, and $e$ is the electronic charge. The integrations extend over the entire energy range. This derivation holds for

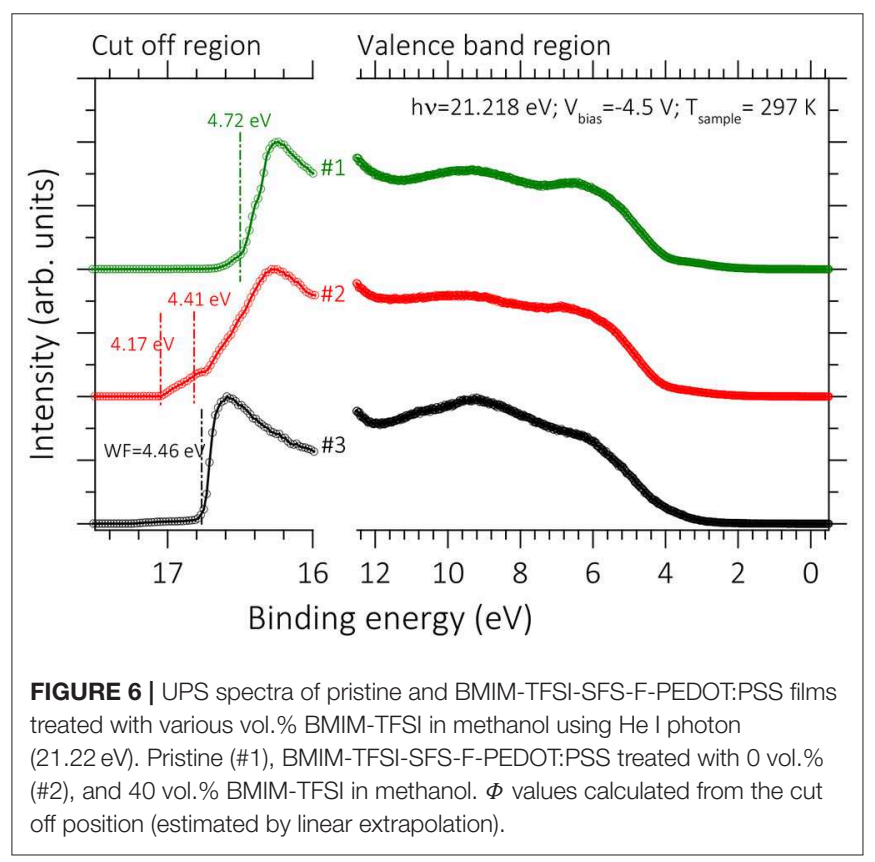

both delocalized and localized states, i.e., band and hopping transport, only the assumption of a Fermi system is necessary. With a further assumption of unipolar charge carrier transport at one narrow transport level $(E \mu)$ Equation (1) provides:

$$
S(T)=\frac{E_{F}(T)-E_{\mu}}{e T}
$$




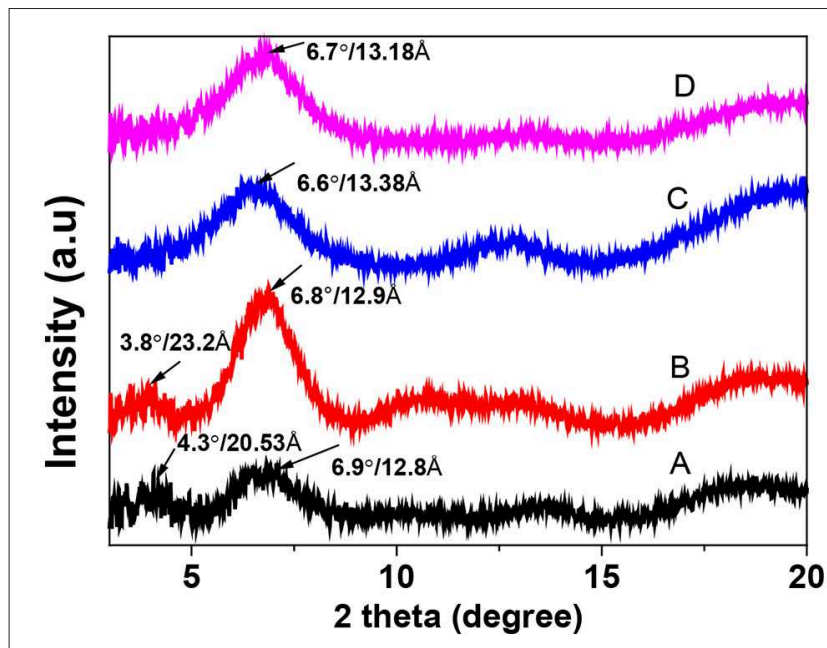

FIGURE $7 \mid X$-ray diffraction (XRD) patterns of pristine and BMIM-TFSI-SFS-F-PEDOT:PSS films treated at various vol.\% BMIM-TFSI in methanol. A refers to Pristine, B refers to F-PEDOT:PSS, C refers to SFS-F-PEDOT:PSS, and D refers to 40 vol.\% BMIM-TFSI-SFS-F-PEDOT:PSS.

In the transport state, the energy difference $E_{F}(T)-E_{\mu}$ is interrelated to the carrier concentration $n(T)$ (Nollau et al., 2000; Sze and Ng, 2006). The variation of about one order decrease of the carrier concentration will increase the $E_{F}(T)-E_{\mu}$, and thus enhance the $S$ according to Equation (2). A similar tendency was observed in doped organic semiconductors between the $n$ and TE properties including the $\sigma$ and $S$ (Nollau et al., 2000). Moreover, as the $S$ relies on the local band structure of the material, the removal of the PSS could transform the local band structure of PEDOT:PSS. Thus, the enhancement in the $S$ may be due to a probable change in the band structure.

The crystallinity of PEDOT:PSS films was studied with the XRD (Figure 7). The pristine film displayed two characteristic peaks at $2 \theta$ values of $4.3^{\circ}$ and $6.9^{\circ}$ corresponding to the lattice $d$ spacing of $20.5 \AA$ and $12.8 \AA$, which were calculated in terms of Bragg's law $(2 d \sin \theta=\lambda)$. They can be assigned to the lamella stacking distance $d(100)$ of the two distinct alternate ordering of PEDOT and the PSS chain. BMIM-TFSI-SFS-F-PEDOT:PSS films showed a slight change from 12.8 to $13.8 \AA$ in the lamella stacking distance of the alternate ordering of PEDOT and the PSS chain (Figure 7). It also displayed relatively sharper diffraction peaks with the higher intensity in the low angle reflections at $2 \theta$ of $6.7^{\circ}$ in comparison with the XRD patterns of the pristine PEDOT:PSS. This corresponded separately to the lamella stacking distance $d$ (100) of two distinct alternate orderings of the PEDOT and the PSS chains, suggesting a higher crystallization degree of the PEDOT:PSS film. Besides, for BMIM-TFSI-SFS-FPEDOT:PSS film, the $2 \theta$ is further shifted to $6.7^{\circ}$ corresponding to a lattice $d$ spacing of $13.8 \AA$, and a fair improvement in diffraction peak intensity was observed. These results demonstrate that the IL treatment makes PEDOT:PSS films to prefer a specific lamella stacking between the PEDOT chains, resulting in the improved crystallinity of the PEDOT:PSS films. The (100) diffraction peak intensity was significantly improved,
TABLE 3 | The thermal properties ( $\rho, C p, b$, and $\kappa$ ) of the pristine and 40 vol. $\%$ BMIM-TFSI-SFS-F-PEDOT:PSS films. The obtained $\kappa$ values were at room temperature.

\begin{tabular}{lcccc}
\hline Code & $\boldsymbol{b}\left(\mathbf{J} / \mathbf{S}^{\mathbf{0 . 5}} \mathbf{m}^{\mathbf{2}}\right)$ & $\boldsymbol{C p} \mathbf{( J / g K )}$ & $\boldsymbol{\rho} \boldsymbol{m}\left(\mathbf{g} / \mathbf{c m}^{\mathbf{3}}\right)$ & $\boldsymbol{\kappa}(\mathbf{W} / \mathbf{m})$ \\
\hline Untreated & 1175 & 1.5 & 1.6 & 0.57 \\
40 vol.\% BMIM- & 785.2 & 1.56 & 1.62 & 0.27 \\
TFSI-SFS-F- & & & & \\
PEDOT:PSS & & & & \\
\hline
\end{tabular}

attributable to the improvement of the number of ordered aggregates associated with interchain $\pi-\pi$ stacking between the PEDOT chain and the enhancement of the crystallinity of the PEDOT:PSS film. Therefore, the XRD results displayed that compared to the pristine films, IL dedoped PEDOT:PSS films showed an enhanced interchain coupling of PEDOT:PSS with a more densely packed PEDOT and lamella stacking between two assemblies, resulting in an improved $S$ of the films through interface scattering (Kim et al., 2014; Wang et al., 2018).

Moreover, the thermal properties of PEDOT:PSS films were investigated and the $\rho, b, C p$, and $\kappa$ of the pristine and BMIMTFSI-SFS-F-PEDOT:PSS films are measured (Table 3). The $\kappa$ of the pristine PEDOT:PSS film was in accordance with those reported by independent groups (Lee et al., 2014a; Wang et al., 2015, 2018). The pulsed light heating thermoreflectance method was used to determine $b$ at room temperature (Baba et al., 2011; Kyaw et al., 2018). The thermalreflectacne signals of the pristine and 40 vol.\% BMIM-TFSI-SFS-F-PEDOT:PSS film after the nanosecond-pulse heating was detected by the probe beam (Figure S3). The effusivity values derived from the curve fitting are given in Table 3. The Cp was obtained using DSC and the $\rho_{m}$ was calculated from the mass and the volume of the film at room temperature. As illustrated in Table 3 the $\kappa$ of BMIMTFSI-SFS-F-PEDOT:PSS films was reduced compared to that of the pristine PEDOT:PSS film. The reduced $\kappa$ could be because of the removal of excess PSS. Therefore, the dimensionless $Z T$ at $300 \mathrm{~K}$ was calculated based on the obtained cross plane $\kappa$ of $0.27 \mathrm{~W} / \mathrm{mK}$ and the corresponding highest $P F$ of 239.2 $\mu \mathrm{W} / \mathrm{K}^{2} \mathrm{~m}$. The $Z T$ value of the BMIM-TFSI-SFS-F-PEDOT:PSS film was $\sim 0.26$ whereas the pristine PEDOT:PSS was $\sim 5.6$ $\times 10^{-6}$ at $300 \mathrm{~K}$. This dramatic enhancement in $Z T$ in the 40 vol.\% BMIM-TFSI-SFS-F-PEDOT:PSS films indicate that our treatment technique is effective for the enhancement of the TE properties of PEDOT:PSS film.

\section{PEDOT:PSS Film Stability Study}

The PEDOT:PSS film stability by measuring the $\sigma, \kappa$, and $S$ of their films at a given humidity and temperature was investigated. The pristine and treated films were put in a humidity chamber at $75 \% \mathrm{RH}$ and $70^{\circ} \mathrm{C}$ for up to $480 \mathrm{~h}$ to investigate the PEDOT:PSS film stability. In this test, 20 batches of samples were prepared and the $\sigma, \kappa$, and $S$ were periodically monitored throughout the stability test. The results of the pristine PEDOT:PSS film were in agreement with the previous reports by the majority of groups on atmospheric exposure of PEDOT:PSS films (Nardes 

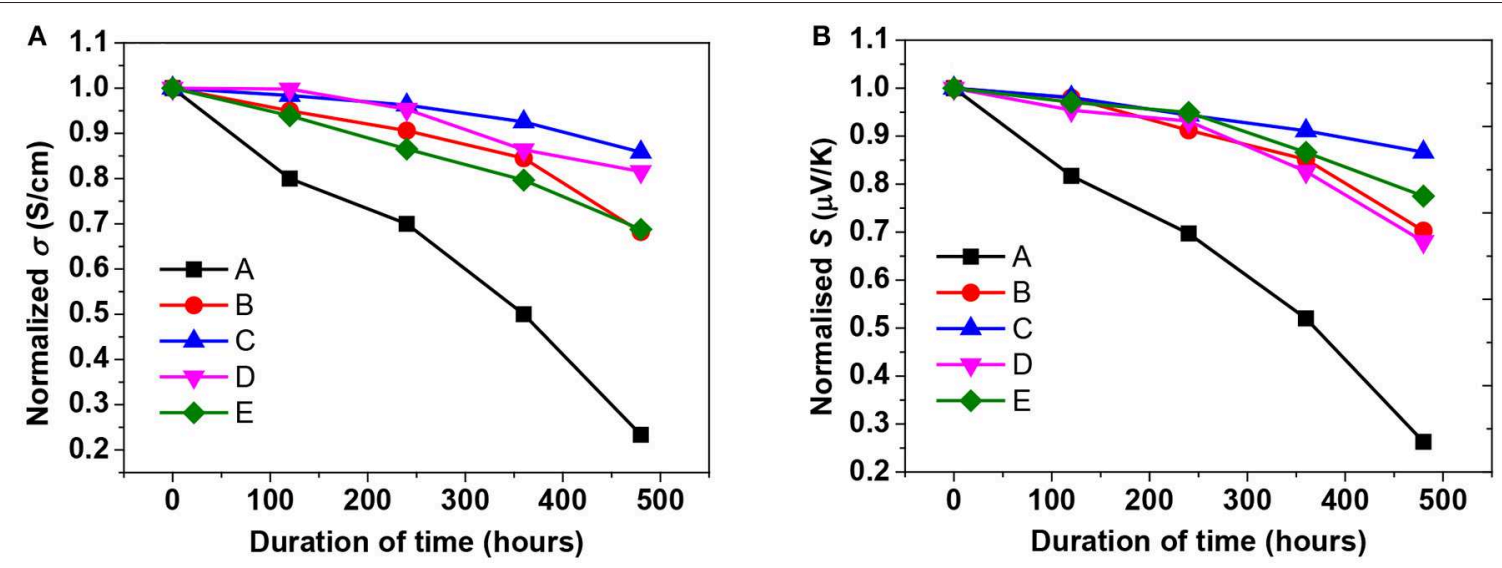

FIGURE 8 | The reduced TE properties of the pristine, formamide treated, and BMIM-TFSI-SFS-F-PEDOT:PSS films treated with various vol.\% BMIM-TFSI in methanol. (A) $\sigma$ and (B) $S$ of the pristine and treated PEDOT:PSS films. Pristine (A), F-PEDOT:PSS (B), SFS-F-PEDOT:PSS (C), 40 vol. \%

BMIM-TFSI-SFS-F-PEDOT:PSS (D), and 60 Vol.\% BMIM-TFSI-SFS-F-PEDOT:PSS (E). Vertical: Si/SO and $\sigma i / \sigma 0$ where $(\sigma 0$ and $S 0)$ and $(S i$ and $\sigma i)$ are $S$ and $\sigma$ at $t=$ $\mathrm{O}$ and the time at which the $S$ and $\sigma$ were recorded respectively, kept over $480 \mathrm{~h}$ under high humidity $(75 \% \mathrm{RH})$ and high temperature $\left(70^{\circ} \mathrm{C}\right)$ conditions.

et al., 2008; Kim et al., 2011; Alemu et al., 2012; McCarthy et al., 2014; Cho et al., 2016). As shown in Figure 8, the $\sigma$ and $S$ of all samples gradually decreased with the increasing exposure time. The relative decrements in the $\sigma$ in the harsh conditions after $480 \mathrm{~h}$ for the pristine PEDOT:PSS film was $77 \%$, while ILs treated PEDOT:PSS films reduced by 22\%, indicating the longterm stability was enhanced through the ILs treatment. It was well noted that the significant reduction in the $\sigma$ of pristine PEDOT:PSS film was owing to the hygroscopic and acidic PSS that picks up water easily (De Jong et al., 2000; Fehse et al., 2008). Since the $\sigma$ deterioration occurred due to absorption of water in the PSS phase (Van Reenen and Kemerink, 2014), the reduction of the PSS in treated PEDOT:PSS film resulted in less water absorption, and subsequently the film was more stable even in a harsh environment. Furthermore, the highly compactstructure brought by the depletion of PSS and the polymer rearrangement may improve the $\sigma$ as well as stability (Nardes et al., 2008). In addition, the stability enhancement may relate to a strong ionic interaction between sulfonate anions and bulky imidazolium cations that blocking the penetration of water into the PEDOT:PSS film and reducing the water uptake. Blending imidazolium derivatives with acidic PEDOT:PSS solutions leads to neutralization of the solution, remarkably enhancing the stability with minimal loss of the $\sigma$; in well agreement with the previous report (Cho et al., 2016).

Furthermore, the cross plane $\kappa$ was observed to be slightly increased for 40 vol.\% BMIM-TFSI-SFS-F-PEDOT:PSS films while the cross-plane $\kappa$ was noticed to be decreased for the pristine PEDOT:PSS film with harsh conditions. The result is in good accordance with a previously observed decline in the elastic modulus of PEDOT:PSS at high humidity conditions (Lang et al., 2009b), i.e., $\kappa=$ (elastic constant) $)^{1 / 2}$ (Hsieh et al., 2011). Hence, the slight increase of $\kappa$ in BMIM-TFSI-SFS-F-PEDOT:PSS films could be due to the film having less PSS and hence less softening conditions and less hygroscopic.
Based on our various observations, we suggest a model for the mechanism of TE properties enhancement for ILsSFS-F-PEDOT:PSS films (Figure 9). Figure 10 also illustrates conformational changes of PEDOT chains for untreated and treated films. Formamide, a polar solvent, with a high dielectric constant induces a strong screening effect between the counter ions and the charge carriers, reducing the interactions between the negatively charged PSS and the positively charged PEDOT. This results in an enlargement of PEDOT chains and the easier removal of PSS due to the change in their conformation from coils to elongated structures (Kim et al., 2002). When SFS $\left(\mathrm{HOCH}_{2} \mathrm{SO}_{2} \mathrm{Na}\right)$ was further introduced into the PEDOT:PSS films, $\mathrm{HOCH}_{2} \mathrm{SO}_{2}^{-}$and $\mathrm{Na}^{+}$ ions penetrated into the PEDOT:PSS film, the $\mathrm{HOCH}_{2} \mathrm{SO}_{2}^{-}$ ions could replace the PSS counter ion and bind to the PEDOT segment as the new counter ion, and the $\mathrm{Na}^{+}$ ions bound to the PSS anions during the treatment. The decreased steric hindrance and dramatically reduced binding effect exerted by PSS led to the conductive PEDOT chain further to elongate and achieve an extended conformation. This extended conformation led to stronger interchain interactions, resulting in a significantly enhanced $\sigma$ of PEDOT:PSS that facilitates charge transport among the polymer chains (Xia and Ouyang, 2011; Culebras et al., 2014). Further addition of ILs e.g. BMIM-TFSI effectively segregated the PEDOT from the PSS, resulting in conformational changes and in turn an increase in mobility. Also, BMIM-TFSI treatment led to electrostatic interactions of the negatively charged PSS with the positively charged BMIM cation and dissociation of the ionic bond between BMIM and TFSI. This led to further elongation of the conductive PEDOT chain to achieve an extended conformation. Figure 10 illustrates conformational changes of the PEDOT chains and a sequential formamide and binary dedoping. This linear-like conformation could change chemical states of PEDOT chains that affect the carrier density in the PEDOT:PSS films (Park et al., 2014b). 


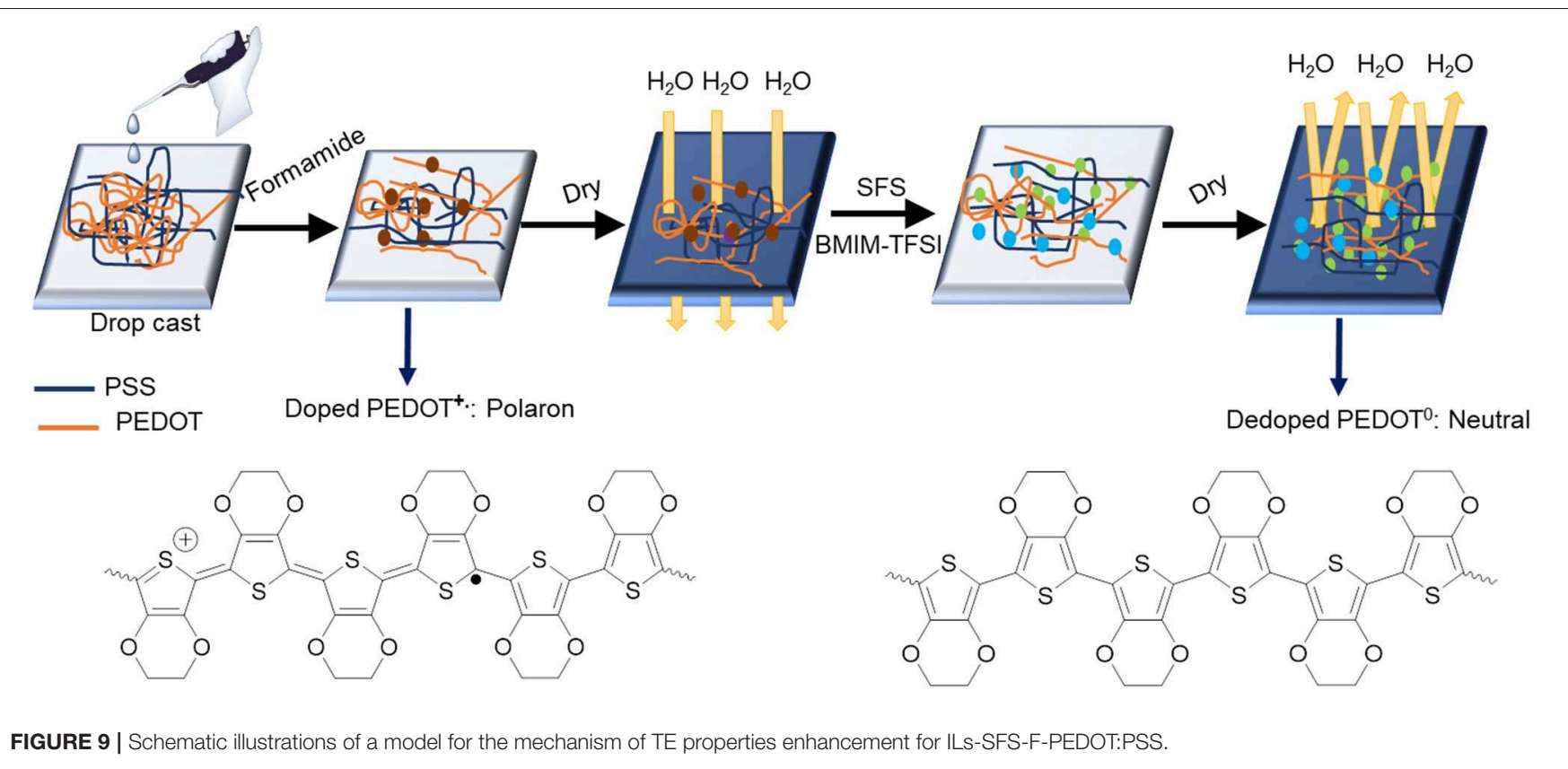

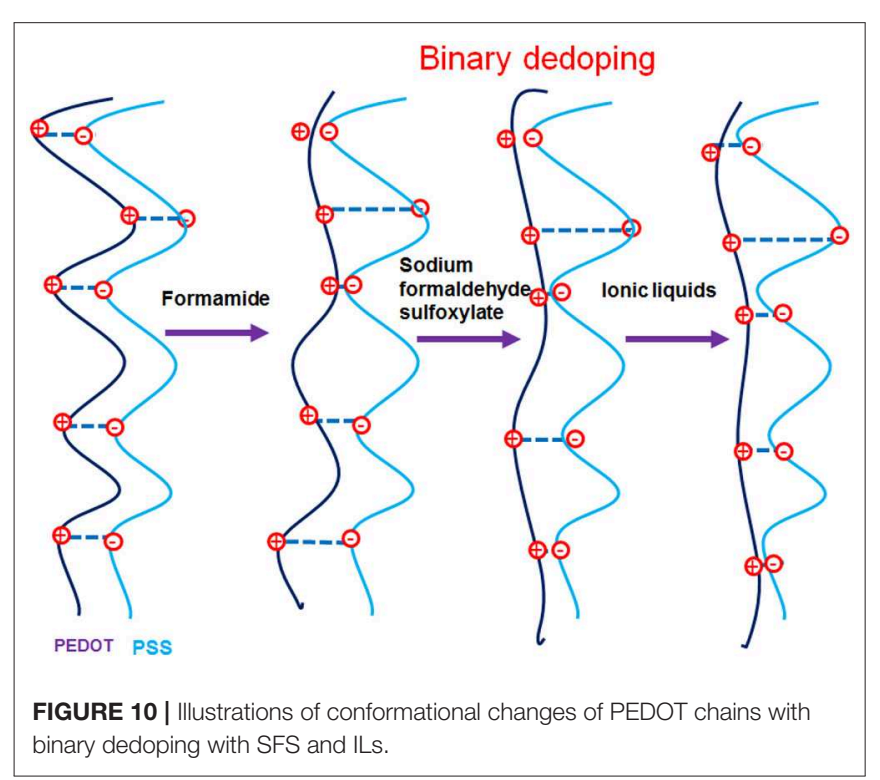

\section{CONCLUSION}

In this study, two series post-treatments using ILs as one of the key steps were reported. Both series post-treatments considerably enhanced TE properties of PEDOT:PSS films. The first series post-treatment resulted in a big jump in the power factor from 63.6 to $137.8 \mu \mathrm{W} / \mathrm{K}^{2} \mathrm{~m}$, corresponding to posttreatment with formamide only, and post-treatment with both formamide and IL, respectively. An additional post-treatment step with SFS was introduced before applying to IL treatment, and the power factor could be further improved to more than $230 \mu \mathrm{W} / \mathrm{K}^{2} \mathrm{~m}$. A large tendency to enhance the power factor likely mainly originated from the improvement in the Seebeck coefficient $S$, which was increased from 14.9 to 28.5 and then $61 \mu \mathrm{V} / \mathrm{K}$ although the electrical conductivity $\sigma$ reduced from a few thousand to in the range of $630-650 \mathrm{~S} / \mathrm{cm}$. Compared to the F-PEDOT:PSS film, the improvement in the $S$ is because of the decrease in the carrier concentration $n$ by roughly $70 \%$ in the case of BMIM-TFSI treated PEDOT:PSS. The enhancement in the $S$ could also be explained by the dedoping process as evidenced by the absorption spectra in which a characteristic absorption peak at $600 \mathrm{~nm}$ appeared. The types of anions associated with ILs also played a role in affecting the magnitude of the power factor in an order of TFSI > OTf $>\mathrm{BF}_{4}$. On the other hand, the cross-plane $\kappa$ reduced from $0.57 \mathrm{~W} / \mathrm{mK}$ for the pristine film to $0.27 \mathrm{~W} / \mathrm{mK}$ for BMIMTFSI-SFS-F-PEDOT:PSS film is largely due to the removal of the PSS. Hence, under the optimum treatment condition, the estimated $Z T$ of $\sim 0.26$ was achieved at $300 \mathrm{~K}$, revealing the potential in the application for harvesting low-grade heat or waste thermal energy.

\section{DATA AVAILABILITY STATEMENT}

All datasets generated for this study are included in the article/Supplementary Material.

\section{AUTHOR CONTRIBUTIONS}

TY, WC, and JX conceived and designed the experiments. TY and $\mathrm{YZ}$ performed the experiments and contributed to the film fabrication, measurement, and data analysis. AK, XW, and 
JS contributed to analysis tools, helped with the analysis, and discussed the data. TY wrote the paper. WC and JX helped revise the paper.

\section{FUNDING}

This work was financed by the Agency for Science, Technology and Research (A*STAR), Industry Alignment Fund, Pharos Hybrid thermoelectric materials for ambient applications Program (Grant Nos. 1527200019 and 1527200021).

\section{REFERENCES}

Alemu, D., Wei, H.-Y., Ho, K.-C., and Chu, C.-W. (2012). Highly conductive PEDOT: PSS electrode by simple film treatment with methanol for ITO-free polymer solar cells. Energy Environ. Sci. 5, 9662-9671. doi: 10.1039/c2ee22595f

Baba, T., Taketoshi, N., and Yagi, T. (2011). Development of ultrafast laser flash methods for measuring thermophysical properties of thin films and boundary thermal resistances. Jpn. J. Appl. Phys. 50:11RA01. doi: 10.1143/JJAP.50.11RA01

Bell, L. E. (2008). Cooling, heating, generating power, and recovering waste heat with thermoelectric systems. Science 321, 1457-1461. doi: 10.1126/science. 1158899

Bubnova, O., Berggren, M., and Crispin, X. (2012). Tuning the thermoelectric properties of conducting polymers in an electrochemical transistor. J. Am. Chem. Soc. 134, 16456-16459. doi: 10.1021/ja305188r

Bubnova, O., Khan, Z. U., Malti, A., Braun, S., Fahlman, M., Berggren, M., et al. (2011). Optimization of the thermoelectric figure of merit in the conducting polymer poly (3, 4-ethylenedioxythiophene). Nat. Mater. 10:429. doi: $10.1038 /$ nmat3012

Chang, C., Wu, M., He, D., Pei, Y., Wu, C.-F., Wu, X., et al. (2018). 3D charge and $2 \mathrm{D}$ phonon transports leading to high out-of-plane ZT in n-type SnSe crystals. Science 360, 778-783. doi: 10.1126/science.aaq1479

Chen, Z., Jian, Z., Li, W., Chang, Y., Ge, B., Hanus, R., et al. (2017). Lattice dislocations enhancing thermoelectric $\mathrm{PbTe}$ in addition to band convergence. Adv. Mater. 29:1606768. doi: 10.1002/adma.201606768

Cho, A., Kim, S., Kim, S., Cho, W., Park, C., Kim, F. S., et al. (2016). Influence of imidazole-based acidity control of PEDOT: PSS on its electrical properties and environmental stability. J. Polym. Sci. B Polym. Phys. 54, 1530-1536. doi: 10.1002/polb. 24048

Chung, T.-C., Kaufman, J., Heeger, A., and Wudl, F. (1984). Charge storage in doped poly (thiophene): optical and electrochemical studies. Phys. Rev. B 30:702. doi: 10.1103/PhysRevB.30.702

Cowen, L. M., Atoyo, J., Carnie, M. J., Baran, D., and Schroeder, B. C. (2017). Organic materials for thermoelectric energy generation. ECS J. Solid State Sci. Technol. 6, N3080-N3088. doi: 10.1149/2.0121703jss

Crispin, X., Jakobsson, F., Crispin, A., Grim, P., Andersson, P., Volodin, A., et al. (2006). The origin of the high conductivity of poly (3, 4-ethylenedioxythiophene)- poly (styrenesulfonate)(PEDOT- PSS) plastic electrodes. Chem. Mater. 18, 4354-4360. doi: 10.1021/cm061032+

Crispin, X., Marciniak, S., Osikowicz, W., Zotti, G., Van Der Gon, A., Louwet, F., et al. (2003). Conductivity, morphology, interfacial chemistry, and stability of poly (3, 4-ethylene dioxythiophene)-poly (styrene sulfonate): a photoelectron spectroscopy study. J. polym. sci. B Polym. Phys. 41, 2561-2583. doi: 10.1002/polb.10659

Culebras, M., Gómez, C., and Cantarero, A. (2014). Enhanced thermoelectric performance of PEDOT with different counter-ions optimized by chemical reduction. J. Mater. Chem. A 2, 10109-10115. doi: 10.1039/C4TA01012D

De Jong, M., Van Ijzendoorn, L., and De Voigt, M. (2000). Stability of the interface between indium-tin-oxide and poly (3, 4-ethylenedioxythiophene)/poly (styrenesulfonate) in polymer light-emitting diodes. Appl. Phys. Lett. 77, 2255-2257. doi: 10.1063/1.1315344

Fan, Z., Du, D., Guan, X., and Ouyang, J. (2018). Polymer films with ultrahigh thermoelectric properties arising from significant seebeck coefficient

\section{ACKNOWLEDGMENTS}

TY would like to thank the SINGA scholarship from the A*STAR Graduate Academy especially. AK would like to thank the startup fund from SUSTech.

\section{SUPPLEMENTARY MATERIAL}

The Supplementary Material for this article can be found online at: https://www.frontiersin.org/articles/10.3389/fchem. 2019.00870/full\#supplementary-material

enhancement by ion accumulation on surface. Nano Energy 51, 481-488. doi: 10.1016/j.nanoen.2018.07.002

Fan, Z., Li, P., Du, D., and Ouyang, J. (2017). Significantly enhanced thermoelectric properties of PEDOT: PSS films through sequential posttreatments with common acids and bases. Adv. Energy Mater. 7:1602116. doi: 10.1002/aenm.201602116

Farah, A. A., Rutledge, S. A., Schaarschmidt, A., Lai, R., Freedman, J. P., and Helmy, A. S. (2012). Conductivity enhancement of poly(3,4ethylenedioxythiophene)-poly(styrenesulfonate) films post-spincasting. J. Appl. Phys. 112:113709. doi: 10.1063/1.4768265

Fehse, K., Meerheim, R., Walzer, K., Leo, K., Lövenich, W., and Elschner, A. (2008). Lifetime of organic light emitting diodes on polymer anodes. Appl. Phys. Lett. 93:312. doi: 10.1063/1.2975369

Fritzsche, H. (1971). A general expression for the thermoelectric power. Solid State Commun. 9, 1813-1815. doi: 10.1016/0038-1098(71)90096-2

Garreau, S., Duvail, J., and Louarn, G. (2001). Spectroelectrochemical studies of poly (3, 4-ethylenedioxythiophene) in aqueous medium. Synth. Met. 125, 325-329. doi: 10.1016/S0379-6779(01)00397-6

Garreau, S., Louarn, G., Buisson, J., Froyer, G., and Lefrant, S. (1999). In situ spectroelectrochemical Raman studies of poly (3, 4-ethylenedioxythiophene)(PEDT). Macromolecules $\quad 32, \quad$ 6807-6812. doi: 10.1021/ma9905674

Greczynski, G., Kugler, T., Keil, M., Osikowicz, W., Fahlman, M., and Salaneck, W. R. (2001). Photoelectron spectroscopy of thin films of PEDOT-PSS conjugated polymer blend: a mini-review and some new results. J. Electron Spectros. Relat. Phenomena 121, 1-17. doi: 10.1016/S0368-2048(01)00323-1

Han, Y. K., Chang, M. Y., Huang, W. Y., Pan, H. Y., Ho, K. S., Hsieh, T. H., et al. (2011). Improved performance of polymer solar cells featuring onedimensional pedot nanorods in a modified buffer layer. J. Electrochem. Soc. 158, K88-K93. doi: 10.1149/1.3534201

Havare, A. K., Can, M., Demic, S., Kus, M., and Icli, S. (2012). The performance of OLEDs based on sorbitol doped PEDOT: PSS. Synth. Met. 161, 2734-2738. doi: 10.1016/j.synthmet.2011.10.011

Hiroshige, Y., Ookawa, M., and Toshima, N. (2007). Thermoelectric figure-of-merit of iodine-doped copolymer of phenylenevinylene with dialkoxyphenylenevinylene. Synth. Met. 157, 467-474. doi: 10.1016/j.synthmet.2007.05.003

Hsieh, W.-P., Losego, M. D., Braun, P. V., Shenogin, S., Keblinski, P., and Cahill, D. G. (2011). Testing the minimum thermal conductivity model for amorphous polymers using high pressure. Phys. Rev. B 83:174205. doi: 10.1103/PhysRevB.83.174205

Hu, Y., Shi, H., Song, H., Liu, C., Xu, J., Zhang, L., et al. (2013). Effects of a proton scavenger on the thermoelectric performance of freestanding polythiophene and its derivative films. Synth. Met. 181, 23-26. doi: 10.1016/j.synthmet.2013.08.006

Huang, J., Miller, P. F., Wilson, J. S., De Mello, A. J., De Mello, J. C., and Bradley, D. D. (2005). Investigation of the effects of doping and post-deposition treatments on the conductivity, morphology, and work function of poly (3, 4ethylenedioxythiophene)/poly (styrene sulfonate) films. Adv. Funct. Mater. 15, 290-296. doi: 10.1002/adfm.200400073

Hwang, J., Amy, F., and Kahn, A. (2006). Spectroscopic study on sputtered PEDOT. PSS: role of surface PSS layer. Org. Electron. 7, 387-396. doi: 10.1016/j.orgel.2006.04.005 
Im, S. G., and Gleason, K. K. (2007). Systematic control of the electrical conductivity of poly (3,4-ethylenedioxythiophene) via oxidative chemical vapor deposition. Macromolecules 40, 6552-6556. doi: 10.1021/ma0628477

Janardhanam, V., Yun, H.-J., Jyothi, I., Lee, J., Hong, H., Reddy, V. R., et al. (2015). Energy-level alignment and electrical properties of Al/p-type Si Schottky diodes with sorbitol-doped PEDOT: PSS as an organic interlayer. J. Alloys Compd. 637, 84-89. doi: 10.1016/j.jallcom.2015.02.210

Jönsson, S., Birgerson, J., Crispin, X., Greczynski, G., Osikowicz, W., Van Der Gon, A. D., et al. (2003). The effects of solvents on the morphology and sheet resistance in poly (3, 4-ethylenedioxythiophene)polystyrenesulfonic acid (PEDOT-PSS) films. Synth. Met. 139, 1-10. doi: 10.1016/S0379-6779(02)01259-6

Kaneko, H., Ishiguro, T., Takahashi, A., and Tsukamoto, J. (1993). Magnetoresistance and thermoelectric power studies of metal-nonmetal transition in iodine-doped polyacetylene. Synth. Met. 57, 4900-4905. doi: 10.1016/0379-6779(93)90836-L

Kemp, N., Kaiser, A., Liu, C., Chapman, B., Mercier, O., Carr, A., et al. (1999). Thermoelectric power and conductivity of different types of polypyrrole. $J$. Polym. Sci. A Polym. Chem. 37, 953-960.

Khan, M., Armes, S., Perruchot, C., Ouamara, H., Chehimi, M., Greaves, S., et al. (2000). Surface characterization of poly (3, 4-ethylenedioxythiophene)coated latexes by X-ray photoelectron spectroscopy. Langmuir 16, 4171-4179. doi: $10.1021 /$ la991390+

Khan, Z. U., Bubnova, O., Jafari, M. J., Brooke, R., Liu, X., Gabrielsson, R., et al. (2015). Acido-basic control of the thermoelectric properties of poly (3, 4ethylenedioxythiophene) tosylate (PEDOT-Tos) thin films. J. Mater. Chem. C 3, 10616-10623. doi: 10.1039/C5TC01952D

Kim, G., Shao, L., Zhang, K., and Pipe, K. P. (2013). Engineered doping of organic semiconductors for enhanced thermoelectric efficiency. Nat. Mater. 12, 719-723. doi: $10.1038 /$ nmat 3635

Kim, G.-H., Kim, J., and Pipe, K. P. (2016). Humidity-dependent thermoelectric properties of poly (3, 4-ethylenedioxythiophene): poly (styrene sulfonate). Appl. Phys. Lett. 108:093301. doi: 10.1063/1.4942598

Kim, J., Jung, J., Lee, D., and Joo, J. (2002). Enhancement of electrical conductivity of poly (3, 4-ethylenedioxythiophene)/poly (4-styrenesulfonate) by a change of solvents. Synth. Met. 126, 311-316. doi: 10.1016/S0379-6779(01) 00576-8

Kim, K. M., Ahn, S., Jang, W., Park, S., Park, O. O., and Wang, D. H. (2018). Work function optimization of vacuum free top-electrode by PEDOT: PSS/PEI interaction for efficient semi-transparent perovskite solar cells. Solar Energy Mater. Solar Cells 176, 435-440. doi: 10.1016/j.solmat.2017.11.002

Kim, N., Kee, S., Lee, S. H., Lee, B. H., Kahng, Y. H., Jo, Y. R., et al. (2014). Highly conductive PEDOT: PSS nanofibrils induced by solution-processed crystallization. Adv. Mater. 26, 2268-2272. doi: 10.1002/adma.201304611

Kim, Y. H., Sachse, C., Machala, M. L., May, C., Müller-Meskamp, L., and Leo, K. (2011). Highly conductive PEDOT: PSS electrode with optimized solvent and thermal post-treatment for ITO-free organic solar cells. Adv. Funct. Mater. 21, 1076-1081. doi: 10.1002/adfm.201002290

Koch, N., Vollmer, A., and Elschner, A. (2007). Influence of water on the work function of conducting poly (3, 4-ethylenedioxythiophene)/poly (styrenesulfonate). Appl. Phys. Lett. 90:043512. doi: 10.1063/1.2435350

Krupka, J. (2013). Contactless methods of conductivity and sheet resistance measurement for semiconductors, conductors and superconductors. Meas. Sci. Technol. 24:062001. doi: 10.1088/0957-0233/24/6/062001

Kyaw, A. K. K., Yemata, T. A., Wang, X., Lim, S. L., Chin, W. S., Hippalgaonkar, K., et al. (2018). Enhanced thermoelectric performance of PEDOT: PSS films by sequential post-treatment with formamide. Macromol. Mater. Eng. 303:1700429. doi: 10.1002/mame.201700429

Lang, U., Müller, E., Naujoks, N., and Dual, J. (2009a). Microscopical investigations of PEDOT: PSS thin films. Adv. Funct. Mater. 19, 1215-1220. doi: $10.1002 / \mathrm{adfm} .200801258$

Lang, U., Naujoks, N., and Dual, J. (2009b). Mechanical characterization of PEDOT: PSS thin films. Synth. Met. 159, 473-479. doi: 10.1016/j.synthmet.2008.11.005

Łapkowski, M., and Pron, A. (2000). Electrochemical oxidation of poly (3, 4-ethylenedioxythiophene)-“in situ" conductivity and spectroscopic investigations. Synth. Met. 110, 79-83. doi: 10.1016/S0379-6779(99)00271-4
Lee, S. H., Park, H., Kim, S., Son, W., Cheong, I. W., and Kim, J. H. (2014a). Transparent and flexible organic semiconductor nanofilms with enhanced thermoelectric efficiency. J. Mater. Chem. A 2, 7288-7294. doi: $10.1039 / C 4 T A 00700 J$

Lee, S. H., Park, H., Son, W., Choi, H. H., and Kim, J. H. (2014b). Novel solutionprocessable, dedoped semiconductors for application in thermoelectric devices. J. Mater. Chem. A 2, 13380-13387. doi: 10.1039/C4TA01839G

Lee, Y. K., Luo, Z., Cho, S. P., Kanatzidis, M. G., and Chung, I. (2019). Surface oxide removal for polycrystalline SnSe reveals near-single-crystal thermoelectric performance. Joule 3, 719-731. doi: 10.1016/j.joule.2019.01.001

Li, J., Tang, X., Li, H., Yan, Y., and Zhang, Q. (2010). Synthesis and thermoelectric properties of hydrochloric acid-doped polyaniline. Synth. Met. 160, 1153-1158. doi: 10.1016/j.synthmet.2010.03.001

Li, J., Zhang, X., Chen, Z., Lin, S., Li, W., Shen, J., et al. (2018). Low-symmetry rhombohedral GeTe thermoelectrics. Joule 2, 976-987. doi: $10.1016 /$ j.joule.2018.02.016

Liang, L., Chen, G., and Guo, C.-Y. (2017). Polypyrrole nanostructures and their thermoelectric performance. Mater. Chem. Front. 1, 380-386. doi: 10.1039/C6QM00061D

Luo, J., Billep, D., Blaudeck, T., Sheremet, E., Rodriguez, R. D., Zahn, D. R., et al. (2014). Chemical post-treatment and thermoelectric properties of poly (3, 4-ethylenedioxylthiophene): poly (styrenesulfonate) thin films. J. Appl. Phys. 115:054908. doi: $10.1063 / 1.4864749$

Luo, J., Billep, D., Waechtler, T., Otto, T., Toader, M., Gordan, O., et al. (2013). Enhancement of the thermoelectric properties of PEDOT: PSS thin films by post-treatment. J. Mater. Chem. A 1, 7576-7583. doi: 10.1039/c3ta11209h

MacDiarmid, A. G. (2001). Synthetic metals: a novel role for organic polymers. Synth. Met. 125, 11-22. doi: 10.1016/S0379-6779(01)00508-2

Mäkinen, A., Hill, I., Shashidhar, R., Nikolov, N., and Kafafi, Z. (2001). Hole injection barriers at polymer anode/small molecule interfaces. Appl. Phys. Lett. 79, 557-559. doi: 10.1063/1.1386400

Mateeva, N., Niculescu, H., Schlenoff, J., and Testardi, L. (1998). Correlation of Seebeck coefficient and electric conductivity in polyaniline and polypyrrole. J. Appl. Phys. 83, 3111-3117. doi: 10.1063/1.367119

McCarthy, J. E., Hanley, C. A., Brennan, L. J., Lambertini, V. G., and Gun'ko, Y. K. (2014). Fabrication of highly transparent and conducting PEDOT: PSS films using a formic acid treatment. J. Mater. Chem. C 2, 764-770. doi: 10.1039/C3TC31951B

Mcgrail, B. T., Sehirlioglu, A., and Pentzer, E. (2015). Polymer composites for thermoelectric applications. Angew. Chem. Int. Ed. 54, 1710-1723. doi: $10.1002 /$ anie.201408431

Moriarty, G. P., Briggs, K., Stevens, B., Yu, C., and Grunlan, J. C. (2013). Fully organic nanocomposites with high thermoelectric power factors by using a dual-stabilizer preparation. Energy Technol. 1, 265-272. doi: 10.1002/ente. 201300018

Na, S.-I., Wang, G., Kim, S.-S., Kim, T.-W., Oh, S.-H., Yu, B.-K., et al. (2009). Evolution of nanomorphology and anisotropic conductivity in solventmodified PEDOT: PSS films for polymeric anodes of polymer solar cells. J. Mater. Chem. 19, 9045-9053. doi: 10.1039/b915756e

Nardes, A. M., Kemerink, M., De Kok, M., Vinken, E., Maturova, K., and Janssen, R. (2008). Conductivity, work function, and environmental stability of PEDOT: PSS thin films treated with sorbitol. Org. Electron. 9, 727-734. doi: 10.1016/j.orgel.2008.05.006

Nollau, A., Pfeiffer, M., Fritz, T., and Leo, K. (2000). Controlled n-type doping of a molecular organic semiconductor: Naphthalenetetracarboxylic dianhydride (NTCDA) doped with bis (ethylenedithio)-tetrathiafulvalene (BEDT-TTF). J. Appl. Phys. 87, 4340-4343. doi: 10.1063/1.373413

Ouyang, J., Chu, C. W., Chen, F. C., Xu, Q., and Yang, Y. (2005). Highconductivity poly (3, 4-ethylenedioxythiophene): poly (styrene sulfonate) film and its application in polymer optoelectronic devices. Adv. Funct. Mater. 15, 203-208. doi: 10.1002/adfm.200400016

Ouyang, J., Xu, Q., Chu, C.-W., Yang, Y., Li, G., and Shinar, J. (2004). On the mechanism of conductivity enhancement in poly $(3,4-$ ethylenedioxythiophene): poly (styrene sulfonate) film through solvent treatment. Polymer 45, 8443-8450. doi: 10.1016/j.polymer.2004.10.001

Park, G. O., Roh, J. W., Kim, J., Lee, K. Y., Jang, B., Lee, K. H., et al. (2014a). Enhanced thermoelectric properties of germanium powder/poly (3, 
4-ethylenedioxythiophene): poly (4-styrenesulfonate) composites. Thin Solid Films 566, 14-18. doi: 10.1016/j.tsf.2014.07.011

Park, H., Lee, S. H., Kim, F. S., Choi, H. H., Cheong, I. W., and Kim, J. H. (2014b). Enhanced thermoelectric properties of PEDOT: PSS nanofilms by a chemical dedoping process. J. Mater. Chem. A 2, 6532-6539. doi: 10.1039/C3TA1 4960A

Park, T., Park, C., Kim, B., Shin, H., and Kim, E. (2013). Flexible PEDOT electrodes with large thermoelectric power factors to generate electricity by the touch of fingertips. Energy Environ. Sci. 6, 788-792. doi: 10.1039/c3ee23729j

Ren, Z. (2017). Thermoelectrics and materials today physics. Elsevier 1, 2-6. doi: 10.1016/j.mtphys.2017.05.001

Saxena, N., Pretzl, B., Lamprecht, X., Bießmann, L., Yang, D., Li, N., et al. (2019). Ionic liquids as Post-treatment agents for simultaneous improvement of seebeck coefficient and electrical conductivity in PEDOT: PSS Films. ACS Appl. Mater. Interfaces 11, 8060-8071. doi: 10.1021/acsami.8b21709

Scott, J., Malliaras, G., Chen, W., Breach, J.-C., Salem, J., Brock, P., et al. (1999). Hole limited recombination in polymer light-emitting diodes. Appl. Phys. Lett. 74, 1510-1512. doi: 10.1063/1.123599

Snaith, H. J., Kenrick, H., Chiesa, M., and Friend, R. H. (2005). Morphological and electronic consequences of modifications to the polymer anode 'PEDOT: PSS'. Polymer 46, 2573-2578. doi: 10.1016/j.polymer.2005. 01.077

Snyder, G. J., and Toberer, E. S. (2011). "Complex thermoelectric materials," in Materials For Sustainable Energy: A Collection of Peer-Reviewed Research and Review Articles from Nature Publishing Group, eds V. Dusastr, J.-M. Tarascon, and M. Gratzel (Pasadena, CA: World Scientific), 101-110. doi: 10.1142/9789814317665_0016

Sze, S. M., and Ng, K. K. (2006). Physics of Semiconductor Devices. Hsinchu; San Jose, CA: John Wiley \& Sons. doi: 10.1002/0470068329

Tan, G., Shi, F., Hao, S., Zhao, L.-D., Chi, H., Zhang, X., et al. (2016). Nonequilibrium processing leads to record high thermoelectric figure of merit in PbTe-SrTe. Nat. Commun. 7:12167. doi: 10.1038/ncomms 12167

Tsai, T.-C., Chang, H.-C., Chen, C.-H., and Whang, W.-T. (2011). Widely variable Seebeck coefficient and enhanced thermoelectric power of PEDOT: PSS films by blending thermal decomposable ammonium formate. Org. Electron. 12, 2159-2164. doi: 10.1016/j.orgel.2011.09.004

Van Reenen, S., and Kemerink, M. (2014). Correcting for contact geometry in Seebeck coefficient measurements of thin film devices. Org. Electron. 15, 2250-2255. doi: 10.1016/j.orgel.2014.06.018

Wang, J., Cai, K., and Shen, S. (2015). A facile chemical reduction approach for effectively tuning thermoelectric properties of PEDOT films. Org. Electron. 17, 151-158. doi: 10.1016/j.orgel.2014.12.007

Wang, X., Kyaw, A. K. K., Yin, C., Wang, F., Zhu, Q., Tang, T., et al. (2018). Enhancement of thermoelectric performance of PEDOT: PSS films by post-treatment with a superacid. RSC $A d v$. 8, 18334-18340. doi: 10.1039/C8RA02058B
Wei, Q., Mukaida, M., Kirihara, K., Naitoh, Y., and Ishida, T. (2015). Recent progress on PEDOT-based thermoelectric materials. Materials 8, 732-750. doi: $10.3390 / \mathrm{ma} 8020732$

Xia, Y., and Ouyang, J. (2011). PEDOT: PSS films with significantly enhanced conductivities induced by preferential solvation with cosolvents and their application in polymer photovoltaic cells. J. Mater. Chem. 21, 4927-4936. doi: $10.1039 / \mathrm{c} 0 \mathrm{jm} 04177 \mathrm{~g}$

Xia, Y., Sun, K., and Ouyang, J. (2012). Highly conductive poly (3, 4ethylenedioxythiophene): poly (styrene sulfonate) films treated with an amphiphilic fluoro compound as the transparent electrode of polymer solar cells. Energy Environ. Sci. 5, 5325-5332. doi: 10.1039/C1EE02475B

Xia, Y. J., and Ouyang, J. Y. (2009). Salt-induced charge screening and significant conductivity enhancement of conducting poly(3,4ethylenedioxythiophene):Poly(styrenesulfonate). Macromolecules 42 4141-4147. doi: 10.1021/ma900327d

$\mathrm{Xu}, \mathrm{K}$., Chen, G., and Qiu, D. (2013). Convenient construction of poly (3, 4-ethylenedioxythiophene)-graphene pie-like structure with enhanced thermoelectric performance. J. Mater. Chem. A 1, 12395-12399. doi: 10.1039/c3ta12691a

Yi, C., Wilhite, A., Zhang, L., Hu, R., Chuang, S. S., Zheng, J., et al. (2015). Enhanced thermoelectric properties of poly (3, 4-ethylenedioxythiophene): poly (styrenesulfonate) by binary secondary dopants. ACS Appl. Mater. Interfaces 7, 8984-8989. doi: 10.1021/acsami.5b01960

Yoo, D., Kim, J., Lee, S. H., Cho, W., Choi, H. H., Kim, F. S., et al. (2015). Effects of one-and two-dimensional carbon hybridization of PEDOT: PSS on the power factor of polymer thermoelectric energy conversion devices. J. Mater. Chem. A 3, 6526-6533. doi: 10.1039/C4TA06710J

Yoon, C., Reghu, M., Moses, D., Cao, Y., and Heeger, A. (1995). Transports in blends of conducting polymers. Synth. Met. 69, 255-258. doi: 10.1016/0379-6779(94)02439-6

Zhang, B., Sun, J., Katz, H., Fang, F., and Opila, R. (2010). Promising thermoelectric properties of commercial PEDOT: PSS materials and their Bi2Te3 powder composites. ACS Appl. Mater. Interfaces 2, 3170-3178. doi: $10.1021 / \mathrm{am} 100654 \mathrm{p}$

Conflict of Interest: The authors declare that the research was conducted in the absence of any commercial or financial relationships that could be construed as a potential conflict of interest.

Copyright (c) 2020 Yemata, Zheng, Kyaw, Wang, Song, Chin and Xu. This is an open-access article distributed under the terms of the Creative Commons Attribution License (CC BY). The use, distribution or reproduction in other forums is permitted, provided the original author(s) and the copyright owner(s) are credited and that the original publication in this journal is cited, in accordance with accepted academic practice. No use, distribution or reproduction is permitted which does not comply with these terms. 